\title{
Minimal forms for function fields of Pfister forms
}




\title{
MINIMAL FORMS FOR FUNCTION FIELDS OF PFISTER FORMS
}

\author{
Detlev W. Hoffmann
}

\begin{abstract}
Let $F$ be a field of characteristic $\neq 2,\left\{\rho_{i}\right\}$ a set of Pfister forms over $F$ and $K=F\left(\left\{\rho_{i}\right\}\right)$ the iterated function field of the $\rho_{i}$ 's over $F$. We investigate the isotropy behaviour of quadratic forms over $F$ under this field extension. ElmanLam-Wadsworth have shown that if the Hasse number $\tilde{u}$ of $F$ is $\leq 2^{n}, n=1,2$, and if all the $\rho_{i}$ are of fold $\geq n$, then $K / F$ is excellent and the Witt kernel $W(K / F)$ is generated by $\left\{\rho_{i}\right\}$. We extend and generalize these results. We show, for example, that if $\tilde{u}(F) \leq 6$ or if $F$ is linked and all the $\rho_{i}$ 's are of fold $\geq 3$, then $K / F$ is excellent. More generally, if $\tilde{u}(F) \leq 2^{n}$ and all the $\rho_{i}^{\prime}$ 's are of fold $\geq n+1$ (resp. $\geq n$ ), then $K / F$ is excellent (resp. $W(K / F)$ is generated by $\left\{\rho_{i}\right\}$ ). We also investigate so-called $K$-minimal forms, i.e. anisotropic forms over $F$ which become isotropic over $K$ but no proper subform does. In two examples, one over $\mathbf{R}(t)$ and one over a formally real global field, we use the methods developed in this paper to compute the $K$-minimal forms explicitly.
\end{abstract}

\section{Introduction}

Let $F$ be a field of characteristic $\neq 2$. In this paper we want to investigate the behaviour of quadratic forms over $F$ over extensions $K / F$ where $K$ is a function field of the form $F\left(\left\{\rho_{i}\right\}\right)$ of Pfister forms $\left\{\rho_{i}\right\}$. We will call such a field extension a Pfister extension. Extensions of this type have been given a thorough treatment by Elman, Lam and Wadsworth in [ELW 1], [ELW 2] and [ELW 3]. They focussed on the following questions: What is the Witt kernel $W(K / F)$ of the ring homomorphism $W F \rightarrow W K$ between the Witt rings of $F$ and $K$ induced by scalar extension? Is the extension $K / F$ excellent, i.e., does there exist to any form $\varphi$ over $F$ a form $\psi$ over $F$ such that the anisotropic part of $\varphi$ over $K$, $\left(\varphi_{K}\right)_{\text {an }}$, is isometric to $\psi_{K}$ ?

Our main motivation will be the determination of so-called $K$-minimal forms for such extensions. These are anisotropic forms over $F$ which become isotropic over $K$ but no proper subforms of them will become isotropic over $K$. Knowing the $K$-minimal forms amounts to knowing which anisotropic forms over $F$ become isotropic over $K$. This problem is related to the excellence problem and to some extent also to the problem of determining $W(K / F)$ as will become clear from our investigations. Thus, it is not surprising that the machinery we develop also leads to some extensions and generalizations of some of the results in the papers mentioned above, most notably [ELW 3].

It is well-known that if $\pi$ is an anisotropic Pfister form over $F$ then $W(F(\pi) / F)=$ $\pi W F$ (see, e.g., [S, Ch.4, Theorem 5.4(iv)]). Thus, it seems natural to ask whether $W(K / F)$ is generated by $\left\{\rho_{i}\right\}$ where $K=F\left(\left\{\rho_{i}\right\}\right)$ is a Pfister extension, i.e.,

$$
W\left(F\left(\left\{\rho_{i}\right\}\right) / F\right)=\sum_{i} \rho_{i} W F .
$$

In [ELW 1], $F$ was called $n$-amenable if equation (1) holds for any finite set $\left\{\rho_{i}\right\}$ of Pfister forms of fold $\leq n$, and $F$ was said to be amenable if $F$ is $n$-amenable for every $n \geq 1$. 
In the context of our paper, it proves useful to extend this definition. We call $F$ co-namenable if equation (1) holds for any finite set $\left\{\rho_{i}\right\}$ of Pfister forms of fold $\geq n$. Thus, $F$ being amenable is the same as $F$ being co-1-amenable. In analogy to the definition of amenability, we say that $F$ is $n$-Pfister excellent (resp. co-n-Pfister excellent) if for every finite set $\left\{\rho_{i}\right\}$ of Pfister forms over $F$ of fold $\leq n$ (resp. $\geq n$ ), the Pfister extension $F\left(\left\{\rho_{i}\right\}\right) / F$ is excellent. We call $F$ Pfister excellent if $F$ is co-1-Pfister excellent (or, equivalently, if $\boldsymbol{F}$ is $\boldsymbol{n}$-Pfister excellent for every $n$ ).

Recall that the Hasse number $\tilde{u}(F)$ of $F$ is defined to be the supremum of the dimensions of all anisotropic forms over $F$ which are indefinite with respect to each ordering on $F$ (if there are any). If $F$ is not formally real (i.e. there are no orderings on $F$ ), then $\tilde{u}(F)$ coincides with the $u$-invariant of $F$, where $u(F)$ (in the generalized sense) is defined to be the supremum of the dimensions of all anisotropic torsion forms over $F$. Let us now state a summary of known "positive" results. In parts (i)-(iv), $\rho, \sigma$, and $\pi$ will denote anisotropic Pfister forms over $F$ with $\operatorname{dim} \rho=2$ and $\operatorname{dim} \sigma=4$.

(i) $W(F(\pi) / F)=\pi W F$ (see above);

(ii) $F(\rho) / F$ and $F(\sigma) / F$ are excellent (see, e.g., [L 1, Ch.VII, Lemma 3.1] or [S, Ch. 2,Lemma 5.1] for $F(\rho)$, and [ELW 1, Appendix II by Arason] for $F(\sigma))$;

(iii) If $\sigma$ is universal then $F(\rho, \sigma) / F$ is excellent (see [ELW 1, Corollary 2.5]);

(iv) $W(F(\rho, \pi) / F)=\rho W F+\pi W F, W(F(\sigma, \pi) / F)=\sigma W F+\pi W F$, and if $\sigma$ is universal then $W(F(\rho, \sigma, \pi) / F)=\rho W F+\sigma W F+\pi W F$. (cf. [ELW 1, Corollary 2.12 and p. 466]);

(v) If $\tilde{u}(F) \leq 2^{n}(n=1,2)$ then $F$ is co-n-amenable and co-n-Pfister excellent (so $F$ is amenable and Pfister excellent if $\tilde{u}(F) \leq 2$ ). Furthermore, if $\tilde{u}(F)=4$ and $F$ is 1-amenable then $F$ is amenable and Pfister excellent (cf. [ELW 3, Main Theorem, Theorem 6.5]).

On the negative side, if $\rho_{1}, \rho_{2}, \rho_{3}$ are 1 -fold Pfister forms then generally $F\left(\rho_{1}, \rho_{2}\right) / F$ is not excellent and $W\left(F\left(\rho_{1}, \rho_{2}, \rho_{3}\right) / F\right) \neq \sum \rho_{i} W F$ (in fact, all biquadratic extensions over $F$ are excellent iff all triquadratic extensions yield the "expected" Witt kernel, but these equivalent conditions generally do not hold, cf. [ELTW]). Thus, fields are neither 1amenable nor 1-Pfister excellent in general. Izhboldin [I] showed recently, that if $\pi$ is an anisotropic $n$-fold Pfister form, $n \geq 3$, then there always exists a field extension $E / F$ such that $E(\pi) / E$ is not excellent. In particular, function fields of $n$-fold Pfister forms, $n \geq 3$, are generally not excellent.

We will extend the above list of positive results. Recall that $F$ is called linked if the quaternion algebras form a subgroup in the Brauer group of $F$. It is well-known that if $F$ is linked then $\tilde{u}(F) \in\{0,1,2,4,8\}$, and all these values do occur (cf. [E]). Also, $F$ is called $n$-linked if any two $n$-fold Pfister forms over $F$ have a common $(n-1)$-fold subform. $F$ is linked iff $F$ is 2-linked.

(vi) Let $n \geq 1$ and let $\tilde{u}(F) \leq 2^{n}$. Then $F$ is co-n-amenable and co- $(n+1)$-Pfister excellent. If $F$ is $(n-1)$-amenable then $F$ is amenable. If $F$ is $n$-Pfister excellent then $F$ is Pfister excellent.

(vii) If $\tilde{u}(F) \leq 6$ or if $F$ is linked, then $F$ is co-3-Pfister excellent.

(viii) Let $n \geq 2$ and let $F$ be an $n$-linked field with $I_{t}^{n+1} F=0$. Suppose that $I^{n} F=$ $J_{n} F$. Then $F$ is co-n-amenable.

These results, especially those in (vi) and (viii), are of interest mainly in the case of 
formally real fields because only then do there exist anisotropic Pfister forms of dimension $>2^{n}$ which can be taken to construct Pfister extensions. If one restricts oneself to function fields of $n$-fold Pfister forms in the above statements about amenability, then these results become a special case of the Linked Group Theorem which we state in the next section.

This paper is structured as follows. In the next section, we will recall several important results which we will need in our proofs. We will also say a little about $(n$-)linked fields and about fields with finite Hasse number. Some familiarity with the basic facts about SAP fields is assumed on the reader's part throughout this paper. The relevant facts can be found in [L 2], [KS], and [ELP]. Many of the proofs consist of rather intricate arguments using signatures of quadratic forms, and very often we will use the fact that certain sets of orderings we define are clopen sets and that $F$ is SAP (which is always guaranteed if $F$ is $n$-linked or the Hasse number is finite). We will not always prove these facts explicitly since they can be readily checked by the reader. In §3, we provide two main lemmas which are somewhat technical in nature but designed in a way so that they can be readily used in the proofs of the results mentioned above and in our investigations of $K$-minimal forms. We will prove above results in $\S 4$. In $\S 5$, we provide bounds for $K$-minimal forms where $K / F$ is a Pfister extension over a field with finite Hasse number. For some of the Pfister extensions $K / F$ we will be dealing with, we will give a characterization of the $K$-minimal forms in $\S 6$. To round things off, we explicitly compute the $K$-minimal forms for certain Pfister extensions $K / F$ in $\S 7$ to demonstrate the power of our approach. In one example, the field $F$ will be a formally real global field, and in the other example $F$ will be $\mathbf{R}(t)$, the rational function field in one variable over the real numbers.

\section{Preliminaries}

By $I^{n} F$ we denote the $n$-th power of the fundamental ideal $I F$ of even-dimensional forms in $W F$. $I^{n} F$ is additively generated by the $n$-fold Pfister forms $\left\langle\left\langle a_{1}, \cdots, a_{n}\right\rangle\right\rangle=\left\langle 1, a_{1}\right\rangle \otimes$ $\cdots \otimes\left\langle 1, a_{n}\right\rangle$. The set of forms over $F$ isometric (resp. similar) to $n$-fold Pfister forms will be denoted by $P_{n} F$ (resp. $G P_{n} F$ ). $P F$ denotes the set of all Pfister forms over $F$ ( $G P F$ is defined accordingly). $W_{t} F$ denotes the torsion part of the Witt ring $W F$, and we put $I_{t}^{n} F=I^{n} F \cap W_{t} F . \quad J_{n} F$ denotes the ideal of forms of degree $\geq n$ in $W F$ as defined by Knebusch in [K 1]. It is conjectured that $I^{n} F=J_{n} F$ for all $n$ and all fields $F$, and it is known to be true in general when $n \leq 4$ (and also for $n=5$ due to some unpublished results by Rost, see [Ka, Théorème 2.8] and the remarks and references there; recently, Voevodsky announced certain results which would imply that this is true for all $n$ ). A generic splitting tower of a form $\varphi$ over $F$ is, in our context, a tower of fields $F=F_{0} \subset F_{1} \subset \cdots \subset F_{h}$ with the $F_{i}$ defined as follows. Let $\varphi_{0} \simeq \varphi_{\text {an }}$, and for $i \geq 1$ let $F_{i}=F_{i-1}\left(\varphi_{i-1}\right)$ and $\varphi_{i} \simeq\left(\left(\varphi_{i-1}\right)_{F_{i}}\right)_{\text {an }} . h \geq 0$ is the smallest integer for which $\operatorname{dim} \varphi_{h} \leq 1$ and $F_{h}$ is called the generic splitting field of $\varphi$ (cf. [K 1] for this construction and other facts about generic splitting towers).

Let $\Phi \subset P_{n} F$ be a set of $n$-fold Pfister forms over $F$. Following the terminology in [ELW 3], we call $\Phi$ a linked group of $n$-fold Pfister forms if $\bar{\Phi}=\left\{\pi \quad\left(\bmod I^{n+1} F\right) \mid \pi \in \Phi\right\}$ is a subgroup of $I^{n} F / I^{n+1} F$. In other words, $\Phi$ is a linked group of $n$-fold Pfister forms if to any $\pi_{1}, \pi_{2} \in \Phi$ there exists $\pi_{3} \in \Phi$ such that $\pi_{1}-\pi_{i} \equiv \pi_{3} \quad\left(\bmod I^{n+1} F\right)$. Furthermore, we say that $\Phi \subset P_{n} F$ generates a linked group of Pfister forms if there exists a linked group $\Psi \subset P_{n} F$ such that $\bar{\Phi}$ generates $\bar{\Psi}$. Recall that two $n$-fold Pfister forms $\pi_{1}, \pi_{2}$ are called linked if there exist an $(n-1)$-fold Pfister form $\rho$ and 1-fold Pfister forms $\tau_{1}, \tau_{2}$ such that $\pi_{i} \simeq \rho \otimes \tau_{i}, i=1,2$. In a linked group of $n$-fold Pfister forms any two Pfister forms 
are linked. The field $F$ is called $n$-linked if $P_{n} F$ is a linked group of Pfister forms. If $n \geq 2$ then it is easy to see that $F$ being $n$-linked implies that $F$ is $m$-linked for all $m \geq n$. If $n=2$ then $F$ is simply called linked.

The next few results show how certain properties of $F$ imply that $F$ is $n$-linked. Here and througout the remainder of the paper, we denote the space of orderings on $F$ by $X_{F}$,

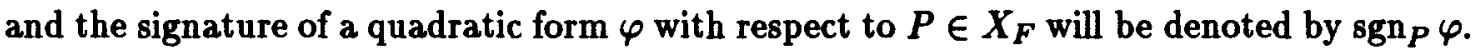
The next lemma is rather obvious and we omit its proof.

Lemma 2.1 Let $\varphi$ be a form over $F$. Then

$$
\operatorname{dim} \varphi_{\text {an }} \leq \sup \left\{\tilde{u}(F),\left|\operatorname{sgn}_{P} \varphi\right| ; P \in X_{F}\right\} .
$$

Lemma 2.2 $F$ is n-linked if one of the following properties holds:

(i) $\tilde{u}(F)<2^{n}+2^{n-1}$.

(ii) $F$ is $S A P$ and $I_{t}^{n} F=0$.

Proof. (i) Let $\pi_{1}, \pi_{2} \in P_{n} F$. If $F$ is formally real then, for all $P \in X_{F}$, we have $\operatorname{sgn}_{P} \pi_{i} \in$ $\left\{0,2^{n}\right\}$ and thus $\left|\operatorname{sgn}_{P}\left(\pi_{1} \perp-\pi_{2}\right)\right| \in\left\{0,2^{n}\right\}$. In any case, the previous lemma implies that $\operatorname{dim}\left(\pi_{1} \perp-\pi_{2}\right)_{\text {an }}<2^{n}+2^{n-1}$. Hence, by [EL 1, Theorem 4.5], $\operatorname{dim}\left(\pi_{1} \perp-\pi_{2}\right)_{\text {an }}=0$ or $2^{n}$ and $\pi_{1}$ and $\pi_{2}$ are linked.

(ii) If $F$ is not formally real then there are no anisotropic $n$-fold Pfister forms and there is nothing to show. So assume that $F$ is formally real and that $\pi_{1}, \pi_{2} \in P_{n} F$. As $F$ is $\operatorname{SAP}$ and as $\left|\operatorname{sgn}_{P}\left(\pi_{1} \perp-\pi_{2}\right)\right| \in\left\{0,2^{n}\right\}$ there exist $\pi \in P_{n} F$ and $x \in \dot{F}$ such that

$$
\operatorname{sgn}_{P} \pi=\left|\operatorname{sgn}_{P}\left(\pi_{1} \perp-\pi_{2}\right)\right| \text { for all } P \in X_{F}
$$

and

$$
\begin{array}{ll}
x<_{P} 0 & \text { for all } P \in X_{F} \text { with } \operatorname{sgn}_{P}\left(\pi_{1} \perp-\pi_{2}\right)=-2^{n} \\
x>_{P} 0 & \text { otherwise. }
\end{array}
$$

Thus, $\operatorname{sgn}_{P}\left(\pi_{1} \perp-\pi_{2}\right)=\operatorname{sgn}_{P} x \pi$ for all $P \in X_{F}$ and therefore

$$
\pi_{1} \perp-\pi_{2} \perp-x \pi \in I_{t}^{n} F=0 .
$$

Hence, $\pi_{1} \perp-\pi_{2}=x \pi$ in $W F$ which shows that $\pi_{1}$ and $\pi_{2}$ are linked.

Example 2.3 Generally, it is not true that $F$ being SAP and $I_{t}^{n} F=0$ implies that $F$ is $(n-1)$-linked. For let $F$ be a field with $\tilde{u}(F)=6$ (such fields exist by Merkurjev's results on the $u$-invariant of fields [M]). Clearly, $F$ is SAP as $\tilde{u}(F)$ is finite, and $I_{t}^{3} F=0$ by the Arason-Pfister Hauptsatz (see below). However, $F$ is not (2-)linked as the Hasse number of a linked field is never 6 (see the remarks in the introduction). As another example, consider the Laurent series field in one variable over the rational numbers, $F=\mathbf{Q}((t))$. Now $u(\mathbf{Q})=4$, and thus, by Springer's theorem (cf. [S, Ch.6, Corollary 2.6]), $u(F)=8$. Hence, $I_{t}^{4} F=0$. On the other hand, $F$ is SAP (cf. [L 2, p. 124]). However, $F$ is not 3 -linked because, for example, $\langle\langle 1,1,1\rangle\rangle$ and $\langle\langle 1,-3, t\rangle\rangle$ are not linked as one can easily check. It will turn out that $F$ is co-4-amenable (see Example 4.3).

For reference reasons, let us now state some well-known and important results which we will use frequently. 
Proposition 2.4 (i) (Cassels-Pfister subform theorem, cf. [S, Ch.4, Theorem 5.4].) Let $\varphi$ be an anisotropic form over $F$ and $\psi$ be another form over $F$. If $\varphi_{F(\psi)}$ is hyperbolic then $a \psi \subset \varphi$ for any $a \in D(\varphi) D(\psi)$. In particular, $\operatorname{dim} \varphi \geq \operatorname{dim} \psi$.

(ii) (Arason-Pfister Hauptsatz (APH), cf. [S, Ch.4, Theorem 5.6].) Let $\varphi$ be an anisotropic form in $I^{n} F$. Then $\operatorname{dim} \varphi \geq 2^{n}$. Furthermore, if $\operatorname{dim} \varphi=2^{n}$ then $\varphi$ is similar to an n-fold Pfister form.

(iii) ("Anisotropy Criterion", [H 4, Theorem 1].) Let $\varphi$ and $\psi$ be forms over $F$ and let $\varphi$ be anisotropic. If $\operatorname{dim} \varphi \leq 2^{n}<\operatorname{dim} \psi$ for some $n$ then $\varphi_{F(\psi)}$ is anisotropic.

(iv) (Extension of orderings, cf. [ELW 2, Theorem 3.5].) Let $F$ be formally real and let $\varphi$ be a form over $F$. Then $P \in X_{F}$ extends to an ordering on $F(\varphi)$ if and only if $\varphi$ is indefinite with respect to $P$, i.e., $\left|\operatorname{sgn}_{P} \varphi\right|<\operatorname{dim} \varphi$. In particular, if $K$ is a generic splitting field as defined above then $P \in X_{F}$ extends to an ordering on $K$ if and only if $\left|\operatorname{sgn}_{P} \varphi\right| \leq 1$.

Here, $a \psi \subset \varphi$ means that $a \psi$ is a subform of $\varphi$, i.e., there exists $\tau \in W F$ such that $\varphi \simeq a \psi \perp \tau$. We will use this notation frequently. Also, $D(\varphi)=\{x \in \dot{F} \mid\langle x\rangle \subset \varphi\}$ denotes the set of non-zero elements in $F$ which are represented by $\varphi$.

The last result we shall mention will play a most crucial role in our investigations. It is the Linked Group Theorem (we will refer to it as LGT) and was proved in [ELW 3, Theorem 2.1].

Theorem 2.5 (Linked Group Theorem.) Let $\left\{\rho_{i}\right\} \subset P_{n} F$ generate a linked group $\Phi$ of Pfister forms over $F$. Let $E / F$ be a field extension and let $L=E\left(\left\{\rho_{i}\right\}\right)$. Then

$$
\Phi \subseteq \sum_{i} \rho_{i} W E \subseteq W(L / E) .
$$

Moreover, if $\psi \in P_{n} E$ then $\psi \in W(L / E)$ if and only if there exists $\rho \in \Phi$ such that $\psi \simeq \rho_{E}$.

Our version here is a touch more general than the original one in which only the case $E=F$ was considered. However, only very minor modifications of the original proof are needed to yield the present form of the theorem.

\section{Two lemmas}

Lemma 3.1 Let $F$ be formally real and $n$-linked. Let $Y \subset X_{F}$ be clopen and let $E / F$ be a field extension such that each $P \in Y$ extends to $E$. Let $\varphi \in W F$ such that $\varphi_{E} \in P_{n} E$. Let $\left\{\pi_{\alpha}\right\} \subset P F$ and suppose that $\varphi_{E\left(\left\{\pi_{\alpha}\right\}\right)}=0$. Then there exists $\pi \in P_{n} F \cap \sum\left(\pi_{\alpha} W F \cap G P_{n} F\right)$ such that $\operatorname{sgn}_{P} \varphi=\operatorname{sgn}_{P} \pi$ for all $P \in Y$.

Proof. If $\varphi_{E}$ is hyperbolic then $\operatorname{sgn}_{P} \varphi=0$ for all $P \in Y$ and we can choose $\pi \in P_{n} F$ to be hyperbolic. So suppose $\varphi_{E}$ is anisotropic. Now $\operatorname{dim} \varphi=2^{n}$ and $\varphi_{E\left(\left\{\pi_{\alpha}\right\}\right)}=0$. Thus, we may assume that $\left\{\pi_{\alpha}\right\}$ is finite and all $\pi_{\alpha}$ are of fold $\leq n$. So let $\left\{\pi_{\alpha}\right\}=$ $\left\{\rho_{1}, \cdots, \rho_{r}, \sigma_{1}, \cdots, \sigma_{s}\right\}$ with $r+s>0, \rho_{i} \in P F$ of fold $\leq n-1$, and $\sigma_{j} \in P_{n} F$. We define a tower of fields $E=L_{0} \subseteq L_{1} \subseteq \cdots \subseteq L_{r}=L \subseteq M$ by $L_{i}=L_{i-1}\left(\rho_{i}\right)$ for $1 \leq i \leq r$ and $M=L\left(\sigma_{1}, \cdots, \sigma_{s}\right)$.

Now $\varphi_{L} \in W(M / L) \cap P_{n} L$. Since $F$ is $n$-linked, LGT implies that there exists $\sigma \in$ $P_{n} F \cap \sum_{i=1}^{s} \sigma_{i} W F$ with $\varphi_{L} \simeq \sigma_{L}$. It is clear by our assumptions that $\operatorname{sgn}_{P} \varphi, \operatorname{sgn}_{P} \sigma \in$ $\left\{0,2^{n}\right\}$ for all $P \in Y$. Let $\psi \simeq \varphi \perp-\sigma$ over $F$. Then $\left|\operatorname{sgn}_{P} \psi\right| \in\left\{0,2^{n}\right\}$ for all $P \in Y$ as well. Since $F$ is SAP (recall that $F$ is $n$-linked!), we can choose $x \in \dot{F}$ such that 
$\operatorname{sgn}_{P} x \psi \in\left\{0,2^{n}\right\}$ for all $P \in Y$. Note also that $\psi_{E} \in W(L / E)$. Let now $Z=\{P \in$ $\left.Y \mid \operatorname{sgn}_{P} x \psi=2^{n}\right\}, Y_{0}=\emptyset$, and for $1 \leq i \leq r$ let

$$
Y_{i}=\left\{P \in Z \backslash\left(Y_{0} \cup \cdots \cup Y_{i-1}\right) \mid \rho_{i} \text { is definite at } P\right\} \text {. }
$$

Then $P \in Z$ extends to $L_{i-1}$ but not to $L_{i}$ iff $P \in Y_{i}$. Since $\psi_{L}=0$, no $P \in Z$ can extend to $L$. Hence, $Z$ is the disjoint union of the $Y_{i}$ 's. Let now $t_{i} \in \dot{F}$ and $\tau_{i} \simeq\left\langle\left\langle 1, \cdots, 1, t_{i}\right\rangle \in P F\right.$ be such that $\tau_{i} \otimes \rho_{i} \in P_{n} F, \tau_{i}$ is definite for all $P \in Y_{i}$ and torsion for all $P \in X_{F} \backslash Y_{i}$. Such $t_{i}$ 's exist as $F$ is SAP and the $Y_{i}$ are clopen sets. One readily checks that $\operatorname{sgn}_{P} \tau_{i} \otimes \rho_{i}=2^{n}$ for all $P \in Y_{i}$ and 0 for all $P \in X_{F} \backslash Y_{i}$. Hence,

$$
\operatorname{sgn}_{P} \sum_{i=1}^{r} \tau_{i} \otimes \rho_{i}= \begin{cases}2^{n} & \text { for } P \in Z \\ 0 & \text { for } P \in X_{F} \backslash Z\end{cases}
$$

and thus $\operatorname{sgn}_{P} \sum_{i=1}^{r} \tau_{i} \otimes \rho_{i}=\operatorname{sgn}_{P} x \psi$ for all $P \in Y$. Hence,

$$
\operatorname{sgn}_{P} \varphi=\operatorname{sgn}_{P}\left(\sigma+\sum_{i=1}^{r} x \tau_{i} \otimes \rho_{i}\right) \text { for all } P \in Y .
$$

Since $F$ is $n$-linked, there exists $\pi \in P_{n} F$ with $\sigma+\sum_{i=1}^{r} x \tau_{i} \otimes \rho_{i} \equiv \pi\left(\bmod I^{n+1} F\right)$. In particular, this yields $\operatorname{sgn}_{P} \varphi \equiv \operatorname{sgn}_{P} \pi\left(\bmod 2^{n+1}\right)$ for all $P \in Y$. In fact, $\operatorname{sgn}_{P} \varphi=$ $\operatorname{sgn}_{P} \pi$ for all $P \in Y$ because $\operatorname{sgn}_{P} \varphi, \operatorname{sgn}_{P} \pi \in\left\{0,2^{n}\right\}$. It is now easy to conclude, using that $F$ is $n$-linked, that $\pi \in \sigma W F+\sum\left(\tau_{i} \otimes \rho_{i}\right) W F$. Moreover, $n$-linkage readily implies that

$$
\pi=\sum_{i=1}^{r} x_{i} \tau_{i} \otimes \rho_{i}+\sum_{j=1}^{s} y_{j} \sigma_{j} \in \sum\left(\pi_{\alpha} W F \cap G P_{n} F\right)
$$

for suitable $x_{i}, y_{j} \in \dot{F}$.

Lemma 3.2 Let $F$ be formally real with $\tilde{u}(F)<2^{n}$ for some $n \geq 1$. Let $\varphi \in W F$ be anisotropic with $\max \left\{\tilde{u}(F), 2^{n-1}\right\}<\operatorname{dim} \varphi \leq 2^{n}$. Let $\left\{\pi_{\alpha}\right\} \subset P F$ be a set of Pfister forms of fold $\geq 1$ and $K=F\left(\left\{\pi_{\alpha}\right\}\right)$. Suppose that $\varphi_{K}$ is isotropic. Then there exists an n-fold Pfister form $\pi \in \sum\left(\pi_{\alpha} W F \cap G P_{n} F\right)$ such that $\varphi$ contains a Pfister neighbor of $\pi$. In particular, there exists $\psi \in W F$ with $\operatorname{dim} \psi<\operatorname{dim} \varphi$ such that $\varphi_{K}=\psi_{K}$ in $W K$.

Proof. Since $\tilde{u}(F)<2^{n}$ we have that $F$ is $n$-linked and SAP. Now let $0 \leq m<2^{n-1}$ such that $\operatorname{dim} \varphi=2^{n}-m$. Since $F$ is $\operatorname{SAP}$, we may assume that after scaling we have $\operatorname{sgn}_{P} \varphi \geq 0$ for all $P \in X_{F}$. Let

$$
\begin{aligned}
& Y_{0}=\left\{P \in X_{F} \mid \operatorname{sgn}_{P} \varphi=\operatorname{dim} \varphi, \text { i.e., } \varphi \text { is definite at } P\right\} \\
& Y_{1}=\left\{P \in X_{F} \mid 0 \leq \operatorname{sgn}_{P} \varphi \leq m\right\}
\end{aligned}
$$

As $F$ is SAP, there exists $\varphi^{\prime} \in W F, \operatorname{dim} \varphi^{\prime}=m$, such that $\operatorname{sgn}_{P} \varphi^{\prime}=m$ (resp. $\operatorname{sgn}_{P} \varphi^{\prime}=$ $-\operatorname{sgn}_{P} \varphi$ ) for all $P \in Y_{0}$ (resp. $P \in Y_{1}$ ). Let $\gamma \simeq \varphi \perp \varphi^{\prime}$. Then $\operatorname{dim} \gamma=2^{n}$ and we have

$$
\operatorname{sgn}_{P} \gamma= \begin{cases}2^{n} & \text { for all } P \in Y_{0} \\ 0 & \text { for all } P \in Y_{1}\end{cases}
$$

Again by the SAP property, there exists $\tau \in P_{n} F$ such that $\operatorname{sgn}_{P} \tau=\operatorname{sgn}_{P} \gamma$ for all $P \in Y_{0} \cup Y_{1}=Y$. Let $E$ be the generic splitting field of $\tau \perp-\gamma$. Since $\tau \perp-\gamma$ has signature 0 at each $P \in Y_{0} \cup Y_{1}=Y$, it follows that each $P \in Y$ extends to $E$ (see 
Proposition 2.4(iv)), and we also have $\tau_{E} \simeq \gamma_{E} \in P_{n} E$. Let $L=E\left(\left\{\pi_{\alpha}\right\}\right)$. Now $\varphi_{K}$ and hence $\gamma_{K}$ is isotropic. Thus, $\gamma_{L} \simeq \tau_{L} \in P_{n} L$ is hyperbolic as $K \subset L$. By Lemma 3.1, there exists $\pi \in \sum\left(\pi_{\alpha} W F \cap G P_{n} F\right)$ such that $\operatorname{sgn}_{P} \gamma=\operatorname{sgn}_{P} \pi$ for all $P \in Y$. Hence, using that $m<\operatorname{sgn}_{P} \varphi<2^{n}-m$ for all $P \in X_{F} \backslash Y$ and that $\operatorname{sgn}_{P} \pi \in\left\{0,2^{n}\right\}$ for all $P$, we get

$$
\left|\operatorname{sgn}_{P}(\varphi \perp-\pi)\right| \leq \begin{cases}m & \text { for all } P \in Y \\ 2^{n}-m-1 & \text { otherwise. }\end{cases}
$$

Now $m<2^{n}-m=\operatorname{dim} \varphi$ and $\tilde{u}(F)<\operatorname{dim} \varphi$ and we get by Lemma 2.1

$$
\operatorname{dim}(\varphi \perp-\pi)_{\text {an }} \leq \max \left\{2^{n}-m-1, \tilde{u}(F)\right\}<\operatorname{dim} \varphi .
$$

Hence, $i_{W}(\varphi \perp-\pi)>\frac{1}{2} \operatorname{dim} \pi$ which implies that $\varphi$ contains a subform of $\pi$ of dimension $\geq 2^{n-1}+1$, i.e., $\varphi$ contains a Pfister neighbor of $\pi$.

The remaining statement of the lemma follows readily by putting $\psi \simeq(\varphi \perp-\pi)_{\text {an }}$ because $\pi_{K}=0$.

\section{Amenability and excellence results}

Theorem 4.1 Let $n \geq 2$. Let $F$ be an $n$-linked field with $I_{t}^{n+1} F=0$. Suppose that $I^{n} F=J_{n} F$. Then $F$ is co-n-amenable.

Proof. Let $\Pi \subset \bigcup_{r>n} P_{r} F$, i.e., $\Pi$ is a set of Pfister forms of fold $\geq n$ over $F$. Let $\Im=\sum_{\pi \in \Pi} \pi W F$. For $m \geq n$ let $\Pi_{m}=\left\{\pi \mid \pi \in \bigcup_{n \leq r \leq m} P_{r} F \cap \Pi\right\}$, i.e. $\Pi_{m}$ consists of all Pfister forms in $\Pi$ of fold $\leq m$. Let $\Im_{m}=\sum_{\pi \in \Pi_{m}} \pi W F$. We obviously have $\Im=\bigcup_{m>n} \Im_{m}$. Let $K=F(I)$. We have to show that $W(K / F)=\Im$.

Clearly, $\Im \subset W(K / F)$. So let $\psi \in W(K / F)$ be anisotropic. Since $K$ is obtained by taking the function field of forms of dimension $\geq 2^{n}$ and since $\psi$ becomes hyperbolic over $K$, it follows from [K 1, Proposition 6.11] that the degree of $\psi$ has to be $\geq n$, i.e. $\psi \in J_{n} F$. By assumption, we then have that $\psi \in I^{n} F$. By [E, Lemma 4.4], there are forms $\alpha_{i} \in P_{i} F$ and $z_{i} \in \dot{F}, n \leq i \leq r$ for some $r \geq n$, such that

$$
\psi=\sum_{i=n}^{r} z_{i} \alpha_{i} \quad \text { in } W F .
$$

It follows immediately from [ELW 1, Lemma 1.3] that $\alpha_{i} \in W(K / F)$ for $n \leq i \leq r$. However, as $\operatorname{dim} \alpha_{i}=2^{i}$ we must already have that $\alpha_{i}$ becomes hyperbolic over $F\left(\Pi_{i}\right)$. Now for $i=n$ we know that $\Pi_{n}$ generates a linked group of Pfister forms because $F$ is $n$-linked. It readily follows from LGT that $\alpha_{n} \in \Im_{n}$.

Now if all $\alpha_{i}=0$ in $W F$ for $i>n$ then we are done. So suppose $i>n$ and $\alpha_{i} \neq 0$. Then $\alpha_{i} \in P_{i} F$ is anisotropic, and because $I_{t}^{i} F=0$ by assumption we necessarily have that $F$ is formally real. Also, $F$ is $i$-linked because it is $n$-linked. Thus, we can apply Lemma 3.1 to conclude that there exists a form $\beta \in \Im_{i} \cap P_{i} F$ such that $\operatorname{sgn}_{P} \alpha_{i}=\operatorname{sgn}_{P} \beta$ for all $P \in X_{F}$. Now the form $\alpha_{i} \perp-\beta$ is in $I^{i} F$ because $\alpha_{i}, \beta \in I^{i} F$, and it has signature 0 at each ordering of $F$. Hence, $\alpha_{i} \perp-\beta \in I_{t}^{i} F=0$. It follows that $\alpha_{i}=\beta$ in $W F$ and thus $\alpha_{i} \in \Im_{i}$. Therefore,

$$
\psi=\sum_{i=n}^{r} z_{i} \alpha_{i} \in \sum_{i=n}^{r} \Im_{i} \subset \Im
$$


Corollary 4.2 Let $n \geq 2$ and suppose that $I^{n} F=J_{n} F$. Then $F$ is co-n-amenable if $F$ satisfies one of the following conditions:

(i) $F$ is $(n-1)$-linked.

(ii) $\tilde{u}(F)<2^{n}+2^{n-1}$.

(iii) $F$ is $S A P$ and $I_{t}^{n} F=0$.

(iv) $F$ is $S A P$ and $u(F)<2^{n}$.

(v) $F$ is n-linked and $u(F)<2^{n+1}$.

In particular, if $F$ satisfies any of these conditions and if $n \leq 4$ then $F$ is co-n-amenable.

Proof. In order to apply the previous theorem we have to verify that $F$ is $n$-linked and $I_{t}^{n+1} F=0$. In (i), this follows from [EL 2, Lemma 2.3, Cor. 2.8]. It is clear that in the remaining cases $I_{t}^{n+1} F=0 . F$ is $n$-linked in (ii) and (iii) by Lemma 2.2. One readily sees that (iv) implies (iii). $F$ is $n$-linked in (v) by assumption. Finally, if $n \leq 4$ then, as mentioned in the introduction, one has $I^{n} F=J_{n} F$ which then implies the last statement.

It is well-known that if $\tilde{u}(F) \leq 2^{n}$ then $I^{n} F=J_{n} F$ (see, e.g., [AK, Lemma 2], or [F, Lemma 4.8]). The above then implies that if $\tilde{u}(F) \leq 2^{n}$ then $F$ is co- $n$-amenable. We do not state this here as another corollary because below we will prove stronger results under the assumption that $\tilde{u}(F) \leq 2^{n}$ (Theorem 4.4 and corollary).

Example 4.3 Let $F=Q((t))$ be the Laurent series field in one variable over the rational numbers. $F$ is easily seen to be 4-linked, the only anisotropic 4-fold Pfister forms being $\langle\langle 1,1,1,1\rangle\rangle$ and $\langle\langle 1,1,1, \pm t\rangle\rangle$. Also, by Springer's theorem, $u(F)=2 u(\mathbf{Q})=8$. By Corollary $4.2, F$ is co-4-amenable. Note that $\tilde{u}(F)=\infty$ as for example the form $\langle 1,-2\rangle \perp(n \times\langle t\rangle)$ is t.i. and anisotropic for each $n$.

Before we state our next main result, we will fix some notation. Let $\left\{\sigma_{i}\right\} \subset P F$ be a set of Pfister forms of fold $\leq n-1$, let $\left\{\rho_{j}\right\} \subset P F$ be a set of Pfister forms of fold $n$, and let $\left\{\pi_{k}\right\} \subset P F$ be a set of Pfister forms of fold $\geq n+1$. Let

$$
\begin{aligned}
& K_{1}=F\left(\left\{\sigma_{i}\right\}\right) \\
& K_{2}=F\left(\left\{\sigma_{i}\right\},\left\{\rho_{j}\right\}\right) \\
& K_{3}=F\left(\left\{\sigma_{i}\right\},\left\{\rho_{j}\right\},\left\{\pi_{k}\right\}\right)
\end{aligned}
$$

and let

$$
\begin{aligned}
& \Im_{1}=\sum_{i} \sigma_{i} W F \\
& \Im_{2}=\sum_{i} \sigma_{i} W F+\sum_{j} \rho_{j} W F \\
& \Im_{3}=\sum_{i} \sigma_{i} W F+\sum_{j} \rho_{j} W F+\sum_{k} \pi_{k} W F .
\end{aligned}
$$

Clearly, $K_{1} \subset K_{2} \subset K_{3}, \Im_{1} \subset \Im_{2} \subset \Im_{3}$, and $\Im_{m} \subset W\left(K_{m} / F\right)$ for $1 \leq m \leq 3$.

Theorem 4.4 Suppose $\tilde{u}(F) \leq 2^{n}$.

(i) If $K_{2} / F$ is excellent then $K_{3} / F$ is excellent.

(ii) If $W\left(K_{1} / F\right)=\Im_{1}$ then $W\left(K_{3} / F\right)=\Im_{3}$.

Proof. (i) We have to show that if $\varphi \in W F$ is anisotropic and $\varphi_{K_{3}}$ is isotropic then $\left(\varphi_{K_{3}}\right)_{\text {an }}$ is defined over $F$.

If $\operatorname{dim} \varphi \leq 2^{n}$, then by the Anisotropy Criterion $\varphi$ must become isotropic already over $K_{2}$. As $K_{2} / F$ is excellent there exists an anisotropic $\mu \in W F$ with $\mu_{K_{2}} \simeq\left(\varphi_{K_{2}}\right)_{\text {an }}$. It 
follows again from the Anisotropy Criterion that $\mu_{K_{2}}$ stays anisotropic over $K_{3}$. Therefore, $\mu_{K_{3}} \simeq\left(\varphi_{K_{3}}\right)_{\text {an }}$ and we are done.

Now if $\operatorname{dim} \varphi>2^{n}$ then by Lemma 3.2 there exists $\psi \in W F$ with $\operatorname{dim} \psi<\operatorname{dim} \varphi$ such that $\varphi_{K_{3}}=\psi_{K_{3}}$ in $W K_{3}$. The assertion now follows easily by using induction on $\operatorname{dim} \varphi$.

(ii) We want to show that $W\left(K_{3} / F\right)=\Im_{3}$. So let $\varphi \in W\left(K_{3} / F\right)$ be anisotropic. If $\operatorname{dim} \varphi<2^{n}$ we already must have $\varphi \in W\left(K_{1} / F\right)=\Im_{1} \subset \Im_{3}$ because anisotropic forms of dimension $<2^{n}$ cannot become hyperbolic over the function field of forms of dimension $\geq 2^{n}$ by the Cassels-Pfister subform theorem. So we are done in this case.

So suppose $\operatorname{dim} \varphi=2^{n}$. If $\operatorname{dim}\left(\varphi_{K_{1}}\right)_{a n}<2^{n}$ we already have $\varphi_{K_{1}}=0$ by the same argument as above. Again, $\varphi \in W\left(K_{1} / F\right)=\Im_{1} \subset \Im_{3}$. So suppose $\operatorname{dim}\left(\varphi_{K_{1}}\right)_{\text {an }}=\operatorname{dim} \varphi=$ $2^{n}$. As $K_{3} / K_{1}$ is obtained by taking function fields of forms of dimension $\geq 2^{n}$, we must have $\operatorname{deg} \varphi_{K_{1}} \geq n$ because $\varphi_{K_{3}}=0$ (see [K 1, Proposition 6.11]). But $\operatorname{dim} \varphi_{K_{1}}=2^{n}$ and thus $\varphi_{K_{1}} \in G P_{n} K_{1}$. After possibly scaling, we may assume that $\varphi$ represents 1 over $F$ and thus over $K_{1}$. Hence, $\varphi_{K_{1}} \in P_{n} K_{1}$. Now $\varphi_{K_{3}}$ is hyperbolic. It is immediate that therefore $\varphi_{K_{2}}$ is already hyperbolic as $K_{3}$ is a function field extension of forms of dimension $\geq 2^{n+1}$ over $K_{2}$. Hence,

$$
\varphi_{K_{1}} \in W\left(K_{2} / K_{1}\right) \cap P_{n} K_{1}
$$

Now $K_{2}=K_{1}\left(\left\{\rho_{j}\right\}\right)$, and as $F$ is $n$-linked because $\tilde{u}(F) \leq 2^{n}$, we have that $\left\{\rho_{j}\right\}$ generates a linked group on $n$-fold Pfister forms over $F$. Thus, by LGT, there exists $\pi \in P_{n} F \cap$ $\left(\sum \rho_{j} W F\right)$ such that $\pi_{K_{1}} \simeq \varphi_{K_{1}}$. In particular,

$$
\varphi \perp-\pi \in W\left(K_{1} / F\right)=\Im_{1} .
$$

Thus, $\varphi \in \Im_{1}+\sum \rho_{j} W F \subset \Im_{3}$.

Now if $\operatorname{dim} \varphi>2^{n}$ and $\varphi_{K_{3}}$ is hyperbolic then, by Lemma 3.2, there exists $\pi \in$ $\Im_{3} \cap G P F$ such that for $\psi \simeq(\varphi \perp-\pi)_{\text {an }}$ we have $\operatorname{dim} \psi<\operatorname{dim} \varphi$. As $\pi \in \Im_{3} \subset W\left(K_{3} / F\right)$ we have $\psi_{K_{3}}=\varphi_{K_{3}} \perp-\pi_{K_{3}}=0$ in $W K_{3}$. Using induction on dimension we conclude that $\psi \in \Im_{3}$ and thus $\varphi \in \Im_{3}$ because $\varphi=\psi \perp \pi$ in $W F$.

Corollary 4.5 Let $\tilde{u}(F) \leq 2^{n}$. Then the following holds.

(i) $F$ is co-( $n+1)$-Pfister excellent. If $F$ is $n$-Pfister excellent then $F$ is Pfister excellent.

(ii) $F$ is co-n-amenable. If $F$ is $(n-1)$-amenable then $F$ is amenable.

We can improve on the above results if we assume that the Hasse number is small enough. These improvements are achieved by invoking well-understood properties of forms of small dimension. In particular, we have the following theorem.

Theorem 4.6 (i) If $\tilde{u}(F) \leq 2$ then $F$ is Pfister excellent.

(ii) If $\tilde{u}(F) \leq 4$ then $F$ is co-2-Pfister excellent.

(iii) If $\tilde{u}(F) \leq 4$ and $F$ is 1-amenable then $F$ is Pfister excellent.

(iv) If $\tilde{u}(F) \leq 6$ then $F$ is co-3-Pfister excellent.

(v) If $F$ is linked then $F$ is co-3-Pfister excellent.

Our proof of parts (i)-(iii) will be shorter than the one given in [ELW 3] although some of our arguments will be in a similar spirit. To prove parts (iv) and (v), we will need the following result. 
Proposition 4.7 Let $\left\{\rho_{i}\right\} \subset P_{3} F$ generate a linked group $\Phi$ of 3-fold Pfister forms over $F$ and let $K=F\left(\left\{\rho_{i}\right\}\right)$. Let $\varphi \in W F$ be anisotropic and suppose that one of the following conditions is satistied.

(i) $\operatorname{dim} \varphi \leq 6$;

(ii) $\operatorname{dim} \varphi=7$, or $\operatorname{dim} \varphi=8$ and $\varphi \in I^{2} F$, and in addition $c(\varphi) \in B r F$ can be represented by a single quaternion algebra, i.e., $c(\varphi)=(x, y)_{F}$ in $B r F$ for some $x, y \in \dot{F}$

(iii) $F$ is linked and $\operatorname{dim} \varphi \leq 8$.

Then $\varphi_{K}$ is isotropic if and only if there exists $\pi \in \Phi$ such that $\varphi$ contains a Pfister neighbor of $\pi$.

Proof. It clearly suffices to show the "only if"-part. By the Anisotropy Criterion we must have $\operatorname{dim} \varphi \geq 5$ for $\varphi_{K}$ to be isotropic. If $\operatorname{dim} \varphi \leq 6$ the above statement was essentially shown in [H 3, Corollary 4.2] in the case of a function field of a single 3-fold Pfister form. The above more general statement can be shown in a similar way after invoking LGT and, at a certain point, the fact that anisotropic 6-dimensional forms in $I^{2}$ stay anisotropic over function fields of forms of dimension $\geq 7$ (see, e.g., [L 3, Theorem 4.3]). We leave it to the reader to fill in the details.

(ii) has been shown in [H 5, Theorem 4.5, Corollary 4.6].

Finally suppose we are in the situation of (iii) and let us assume that $\varphi_{K}$ is isotropic. If $\operatorname{dim} \varphi \leq 6$ we are done by part (i). So suppose $\operatorname{dim} \varphi=7$ or 8 . As $F$ is linked there exist $x, y \in \dot{F}$ such that $c(\varphi)=(x, y)_{F} \in B r F$. If $\operatorname{dim} \varphi=7$, or if $\operatorname{dim} \varphi=8$ and $\varphi \in I^{2} F$ the result then follows from (ii). So let us assume that $\operatorname{dim} \varphi=8$ and that $d=d_{ \pm} \varphi \neq 1 \in \dot{F} / \dot{F}^{2}$. Let $E=F(\sqrt{d})$. Suppose that $\varphi_{E}$ is isotropic. Then there exist $z \in \dot{F}$ and $\alpha \in W F$ such that $\varphi \simeq \alpha \perp z\langle 1,-d\rangle$. This yields $d_{ \pm} \alpha=1$, i.e., $\alpha$ is a 6-dimensional form in $I^{2} F$. But over a linked field 6-dimensional $I^{2}$-forms are always isotropic, a contradiction to $\varphi$ being anisotropic. Hence, $\varphi_{E}$ is anisotropic.

Let $L=E(\langle\langle-x,-y\rangle\rangle)$. If $\langle\langle-x,-y\rangle\rangle_{E}$ is isotropic then $L / E$ is purely transcendental and $\varphi$ stays anisotropic over $L$. If $\left\langle\langle-x,-y\rangle_{E}\right.$ is anisotropic then obviously $\left\langle\langle-x,-y\rangle_{L}=0\right.$ or, equivalently, $(x, y)_{L}=1$ in $\operatorname{BrL}$. Thus, $\varphi_{L} \in I^{3} L$, and as $\operatorname{dim} \varphi=8$ we have $\varphi_{L} \in$ $G P_{3} L$. Suppose $\varphi_{L}$ is isotropic. Then in fact $\varphi_{L}=0$, i.e., $\varphi_{E} \in W(L / E)$ and there exists a form $\tau \in W E, \operatorname{dim} \tau=2$, such that $\varphi_{E} \simeq \tau \otimes\langle\langle-x,-y\rangle\rangle_{E}$. In particular, $\varphi_{E} \in G P_{3} E$ and thus $c\left(\varphi_{E}\right)=1=(x, y)_{E}$ in $B r E$, which in turn implies that $\langle(-x,-y\rangle\rangle_{E}=0$, a contradiction. Hence, $\varphi_{L} \in G P_{3} L$ is anisotropic. We may assume that $\varphi$ represents 1 over $F$ and thus, $\varphi_{L} \in P_{3} L$. Now clearly $\varphi_{L}$ becomes isotropic and hence hyperbolic over $L\left(\left\{\rho_{i}\right\}\right)$. By LGT, there exists $\pi \in \Phi$ such that $\varphi_{L} \simeq \pi_{L}$. As $F$ is linked we have $\tilde{u}(F) \leq 8$ (cf. [E, Theorem 4.7]). As $F$ is SAP there exists $a \in \dot{F}$ such that $\left|\operatorname{sgn}_{P}(\varphi \perp-a \pi)\right| \leq 8$ for all $P \in X_{F}$. Let $\psi \simeq(\varphi \perp-a \pi)_{\text {an }}$. We then have $\operatorname{dim} \psi \leq \max \left\{\tilde{u}(F), \mid \operatorname{sgn}_{P}(\varphi \perp\right.$ $\left.-a \pi) \mid ; P \in X_{F}\right\} \leq 8$.

Suppose $\operatorname{dim} \psi=8$. We have $d_{ \pm} \psi=d_{ \pm}(\varphi \perp-\pi)=d_{ \pm} \varphi=d$ and $c(\psi)=c(\varphi \perp-\pi)=$ $c(\varphi)=(x, y)_{F}$. By the same argument as before, we see that $\psi_{L}$ is anisotropic. But

$$
\psi_{L}=\varphi_{L} \perp-a \pi_{L}=\pi_{L} \perp-a \pi_{L} \quad \text { in } W L .
$$

Comparing dimensions, we see that $\pi_{L} \perp-a \pi_{L} \in P_{4} L$ is isotropic and hence hyperbolic, which in turn implies that $\psi_{L}=0$ in $W L$, a contradiction. Hence, $\operatorname{dim} \psi<8$, i.e., $\operatorname{dim} \psi \leq 6$. Then we have $i_{W}(\varphi \perp-a \pi) \geq 5$ and there exists $\chi \in W F$ with $\operatorname{dim} \chi=5$, $\chi \subset \varphi$ and $\chi \subset a \pi$, which concludes the proof. 
An analogous result to that in (ii) also holds if $\varphi \in I^{2} F, \operatorname{dim} \varphi=8$, and $c(\varphi)=$ $(x, y)_{F} \otimes(u, v)_{F}$ in $B r F$, where $x, y, u, v \in \dot{F}$ (i.e., $c(\varphi)$ can be represented by a biquaternion algebra in $B r F$, or equivalently, ind $c(\varphi) \leq 4)$. This follows easily from [Lag, Théorème 2(iv)] after a minor modification of the proof given there. If $\varphi \in I^{2} F$ and $\operatorname{dim} \varphi=8$ then one easily shows that ind $c(\varphi) \leq 8$, and if it is 8 then $\varphi$ is necessarily anisotropic and it follows from Merkurjev's index reduction theorem [M] that $\varphi$ will stay anisotropic over function fields of 3 -fold Pfister forms. If $\operatorname{dim} \varphi=8$ and $d_{ \pm} \varphi \neq 1$, then the above conclusion generally fails to be true as follows from Izhboldin's results in [I], see also [H 6].

Proof of Theorem 4.6. Let $n=1$ in (i), $n=2$ in (ii) and (iii), and $n=3$ in (iv) and (v). We then have $\tilde{u}(F) \leq 2^{n}$. By the definition of (co-n) Pfister excellence and after invoking Theorem 4.4 it suffices to show that if $\left\{\rho_{i}\right\} \subset P_{n} F$ (resp. $\left\{\rho_{i}\right\} \subset P_{1} F \cup P_{2} F$ in part (iii)) and if $K=F\left(\left\{\rho_{i}\right\}\right)$ then $K / F$ is excellent. To show this, let $\varphi \in W F$ be anisotropic and suppose that $\varphi_{K}$ is isotropic. If $\operatorname{dim} \varphi>\tilde{u}(F)$ then, by Lemma 3.2, there exists $\psi \in W F$ with $\operatorname{dim} \psi<\operatorname{dim} \varphi$ such that $\psi_{K}=\varphi_{K}$ in $W K$. Hence, it suffices to show that if $\varphi \in W F$ is anisotropic with $\operatorname{dim} \varphi \leq \tilde{u}(F)$, and if $\varphi_{K}$ is isotropic then there exists $\psi \in W F$ with $\operatorname{dim} \psi<\operatorname{dim} \varphi$ such that $\psi_{K}=\varphi_{K}$ in $W K$. This then establishes the excellence of $K / F$.

Now if $\operatorname{dim} \varphi \leq 3$ or $\varphi \in G P F$ then for any field extension $K / F$ we have that $\left(\varphi_{K}\right)_{\text {an }}$ is defined over $F$. We thus are done with (i). To establish (ii) (resp. (iii)), it suffices to consider an anisotropic form $\varphi \simeq\langle d, a, b, a b\rangle \in W F$ with $\varphi_{K}$ isotropic and show that $\left(\varphi_{K}\right)_{\text {an }}$ is defined over $F$. If $\varphi_{K}=0$ there is nothing else to show, so we may assume $d \notin \dot{K}^{2}$. First, consider (ii). Since $\tilde{u}(F) \leq 4$, the $\left\{\rho_{i}\right\} \subset P_{2} F$ form a linked group $\Phi$ of 2-fold Pfister forms. Now over $E=F(\sqrt{d})$ we have $\varphi_{E} \in P_{2} E$. Clearly, $\varphi$ becomes isotropic and hence hyperbolic over $E\left(\left\{\rho_{i}\right\}\right.$ and by LGT there exists $\rho \simeq\langle\langle u, v\rangle\rangle \in \Phi$ such that $\varphi_{E} \simeq \rho_{E}$. But then also $\varphi_{E} \simeq\langle d, a, b, a b\rangle_{E} \simeq\langle d, u, v, u v\rangle_{E}$ and by [W, Theorem 7] it follows that $\varphi$ and $\langle d, a, b, a b\rangle$ are similar over $F$. In particular, $\varphi$ contains a subform similar to $\langle u, v, u v\rangle$ which is a Pfister neighbor of $\rho \in \Phi \subset W(K / F)$. Thus, for suitable $x \in \dot{F}$ and with $\psi \simeq(\varphi \perp x \rho)_{\text {an }}$ we get $\operatorname{dim} \psi=2<\operatorname{dim} \varphi$ and $\psi_{K}=\varphi_{K} \perp x \rho_{K}=\varphi_{K}$ in $W K$, and we are done.

To prove (iii), let $\varphi \simeq\langle d, a, b, a b\rangle$ be as above with $\varphi_{K}$ isotropic but not hyperbolic, so that again $d \notin \dot{K}^{2}$ and $\varphi_{K}$ is similar to $\langle 1,-d\rangle_{K}$. Over $L=K(\langle 1,-d\rangle)=F\left(\left\{\rho_{i}\right\},\langle 1,-d\rangle\right)$ we have $\varphi_{L}=0$. We know from Corollary 4.5 that $F$ is amenable. Thus, there are $\tau, \tau_{i} \in W F$ such that $\varphi=\sum_{i} \tau_{i} \otimes \rho_{i}+\tau \otimes\langle 1,-d\rangle$ in $W F$. Now over $K$ we have $\varphi_{K}=$ $\tau_{K} \otimes\langle 1,-d\rangle_{K}$ in $W K$, and as $d \notin \dot{K}^{2}$ we must have that $\operatorname{dim} \tau$ is odd. Choose $b \in \dot{F}$ such that $\tau \perp\langle-b\rangle \in I^{2} F$. We then get that $(\tau \perp\langle-b\rangle) \otimes\langle 1,-d\rangle \in I^{3} F$. Thus,

$$
(\varphi \perp-b\langle 1,-d\rangle)_{K}=(\tau \perp\langle-b\rangle)_{K} \otimes\langle 1,-d\rangle_{K} \in I^{3} K
$$

But $\operatorname{dim}(\varphi \perp-b\langle 1,-d\rangle)=6$ and therefore, APH implies that $(\varphi \perp-b\langle 1,-d\rangle)_{K}=0$ and thus $\varphi_{K}=b\langle 1,-d\rangle_{K}$ in $W K$. (iii) now follows.

If $\tilde{u}(F) \leq 6$ then $F$ is 3 -linked and therefore $\left\{\rho_{i}\right\} \subset P_{3} F$ generates a linked group $\Phi$ of 3-fold Pfister forms. In this case, it suffices to consider the case $\operatorname{dim} \varphi \leq 6$. By invoking Proposition 4.7(i), we can conclude that there exists $\pi \in W(K / F) \cap G P_{3} F$ such that $\psi \simeq(\varphi \perp-\pi)_{\text {an }}$ has dimension $\leq 4$. But then $\psi_{K}$ stays anisotropic (its dimension is $\leq 4$ and $K$ is a function field of forms of dimension 8 !). Clearly, $\psi_{K}=\varphi_{K}$ and the anisotropic part of $\varphi_{K}$ is therefore defined over $F$ by $\psi_{K}$. This establishes (iv).

In (v), excellence of $K / F$ follows in a similar manner from Proposition 4.7(iii). 
Corollary 4.8 Let $F$ be a field such that $\tilde{u}(F) \leq 6$ or $F$ is linked. Then $F(\pi) / F$ is excellent for any Pfister form $\pi$ over $F$.

\section{$5 \quad K$-minimal forms}

Let $K / F$ be a field extension. In [H 1], the notion of a $K$-minimal form has been introduced. A form $\varphi$ over $F$ is said to be $K$-minimal if $\varphi$ is anisotropic, $\varphi_{K}$ is isotropic, and $\eta_{K}$ is anisotropic for any subform $\eta \subset \varphi$ with $\operatorname{dim} \eta<\operatorname{dim} \varphi$. In general, the characterization of $K$-minimal forms seems to be a rather difficult problem so that it is already quite desireable just to have some information on bounds for the dimensions of $K$-minimal forms. This leads to the definition of the following two invariants $t_{\max }$ and $t_{\min }$ of the field extension $K / F$ which have been introduced in [H1] :

$$
\begin{aligned}
& t_{\max }(K / F)=\sup \{\operatorname{dim} \varphi \mid \varphi \in W F \text { is } K \text {-minimal }\} \\
& t_{\min }(K / F)=\min \{\operatorname{dim} \varphi \mid \varphi \in W F \text { is } K \text {-minimal }\}
\end{aligned}
$$

We put $t_{\min }(K / F)=t_{\max }(K / F)=1$ if there are no $K$-minimal forms, i.e., if all anisotropic forms over $F$ stay anisotropic over $K$.

Our interest is focused on the case where $K / F$ is a Pfister extension. As an easy example, let $\rho \simeq\langle 1,-d\rangle \in P_{1} F$ be anisotropic and let $K=F(\rho)=F(\sqrt{d})$. Then the $K$-minimal forms over $F$ are exactly the forms similar to $\rho$. In particular, $t_{\min }(K / F)=$ $t_{\max }(K / F)=2($ see $[\mathrm{L} 1, \mathrm{Ch} . \mathrm{VII}, 3.1]$, or [S, Ch.2, 5.1]). The situation is considerably more complicated in the case where $K=F(\rho)$ with an anisotropic $\rho \in P_{2} F$. A characterization of $K$-minimal forms in this case can be found in [HLVG], and in [HVG] it has been dealt with the question how properties of $F$ might affect $t_{\max }(K / F)$. In particular, an example of a field $F$ and an anisotropic $\rho \in P_{2} F$ has been constructed such that $t_{\max }(K / F)=\infty$.

Generally, very little is known regarding $K$-minimal forms when $K=F(\rho)$ for an anisotropic $\rho \in P_{n} F$ if $n \geq 3$. In these cases, we do not even have that $K / F$ is excellent in general. In [I], beside these non-excellence results one can also find examples of such $F$ and $\rho$ where there exist $2^{n}$-dimensional $K$-minimal forms. These examples have been generalized in [H 6].

We do have the following.

Theorem 5.1 Let $\emptyset \neq\left\{\rho_{i}\right\} \subset P_{n} F$ be a set of anisotropic Pfister forms and let $L=$ $F\left(\left\{\rho_{i}\right\}\right)$. Let $K / L$ with $t_{\min }(K / L)=1$ or $t_{\min }(K / L)>2^{n-1}+1$. Then $t_{\min }(K / F)=$ $2^{n-1}+1$. Furthermore, if the set $\left\{\rho_{i}\right\}$ generates a linked group $\Phi$ of $n$-fold Pfister forms then an anisotropic form $\varphi \in W F$ with $\operatorname{dim} \varphi=2^{n-1}+1$ is $K$-minimal iff $\varphi$ is a Pfister neighbor of some $\rho \in \Phi$. In particular, if $\left\{\rho_{i}\right\}=\{\rho\}$ is a singelton set then the $K$-minimal forms of dimension $2^{n-1}+1$ are exactly the Pfister neighbors of $\rho$ of dimension $2^{n-1}+1$.

Proof. Any anisotropic form $\varphi$ over $F$ of dimension $\leq 2^{n-1}+1$ which becomes isotropic over $K$ must already becomes isotropic over $L$. By the Anisotropy Criterion we have $t_{\min }(L / F) \geq 2^{n-1}+1$ and thus $t_{\min }(K / F) \geq 2^{n-1}+1$. However, clearly any Pfister neighbor of dimension $2^{n-1}+1$ of any Pfister form in $\left\{\rho_{i}\right\}$ becomes isotropic over $K$. Hence, $t_{\min }(K / F)=2^{n-1}+1$.

To show the second part of the theorem, it suffices to show that if $\varphi \in W F$ is anisotropic and $\operatorname{dim} \varphi=2^{n-1}+1$, and if $\varphi_{K}$ is isotropic, then $\varphi$ is a Pfister neighbor of some $\rho \in \Phi$. So let $\varphi$ be as above. By the above, $\varphi$ already becomes isotropic over $L=F\left(\left\{\rho_{i}\right\}\right)$. In $\left[H 4\right.$, Theorem 3], it was shown that if $\left\{\rho_{i}\right\}=\{\rho\}$ is a singelton set then $\varphi_{L}$ being isotropic implies that $\varphi$ is a Pfister neighbor of $\rho$. The proof of this statement given in [H 4] readily 
carries over to the general case using the assumption that the set $\left\{\rho_{i}\right\}$ generates a linked group of Pfister forms and then invoking LGT. This then leads to the desired conclusion that there exists $\rho \in \Phi$ such that $\varphi$ is a Pfister neighbor of $\rho$. We omit the details.

This theorem applies in particular to the situation where $K=F\left(\left\{\rho_{i}\right\},\left\{\pi_{j}\right\}\right)$ with $\left\{\pi_{j}\right\} \subset P F$ a set of anisotropic Pfister forms of fold $>n$. In this case, $K=L\left(\left\{\pi_{j}\right\}\right)$ and the Anisotropy Criterion implies that either $t_{\min }(K / L)=1$ or $t_{\min }(K / L)>2^{n}$.

Remark 5.2 Suppose the $\rho_{i}$ 's in the above theorem generate a linked group of Pfister forms. If $n=2$, one can show that there are no 4-dimensional minimal forms (this is implicit in our proof of Theorem 4.6(ii)), and if $n=3$, it follows from Proposition 4.7 that there are no 6-dimensional minimal forms. But as mentioned above, there can be 8-dimensional minimal forms.

The next theorem provides upper bounds for a Pfister extension $K / F$ under the additional assumption that $\tilde{u}(F)<\infty$.

Theorem 5.3 Let $k \geq 1$ and let $\left\{\pi_{\alpha}\right\} \subset P F$ be a set of anisotropic Pfister forms of dimension $\leq 2^{k}$ such that $\left\{\pi_{\alpha}\right\} \cap P_{k} F \neq \emptyset$. Let $K=F\left(\left\{\pi_{\alpha}\right\}\right)$. Then $t_{\max }(K / F) \leq$ $\max \left\{\tilde{u}(F), 2^{k}+1\right\}$.

Furthermore, if $\left\{\pi_{\alpha}\right\} \cap P_{k} F=\{\rho\}$ is a singleton set then $t_{\max }(K / F) \leq \max \{\tilde{u}(F)$, $\left.2^{k-1}+1\right\}$.

Proof. We clearly may assume that $\tilde{u}(F)<\infty$, say $\tilde{u}(F) \leq 2^{\ell}$ for some $\ell$. We also may assume that $F$ is formally real because otherwise there are no anisotropic forms over $F$ of dimension $>\tilde{u}(F)$.

Let $\varphi \in W F$ be anisotropic with $\operatorname{dim} \varphi>\tilde{u}(F)$ and $\varphi_{K}$ isotropic. Then by Lemma 3.2, $\varphi$ contains a Pfister neighbor $\gamma$ of dimension $2^{n-1}+1$ of some $\pi \in P_{n} F \cap W(K / F)$ for some $n \geq 1$. Obviously, $\gamma_{K}$ is isotropic because $\pi_{K}=0$. To show the first part of the theorem, it therefore suffices to show that if $n-1>\max \{k, \ell-1\}$ then $\gamma$ is not $K$-minimal.

So let $n-1>\max \{k, \ell-1\}$, let $\pi \in P_{n} F \cap W(K / F)$ be anisotropic and let $\gamma$ be a Pfister neighbor of $\pi$. As $\tilde{u}(F)<\infty$ we have that $F$ is SAP, so we may assume that after scaling we have $\operatorname{sgn}_{P} \gamma \geq 1$ for all $P \in X_{F}$ (note that $\operatorname{dim} \gamma$ is odd because $n-1 \geq 1$ ). By Lemmas 3.1 and 3.2, there exist an integer $r>0, t_{i} \in \dot{F}, \rho_{i} \in\left\{\pi_{\alpha}\right\}, 1 \leq i \leq r$, such that for $\tau_{i} \simeq\left\langle\left\langle 1, \ldots, 1, t_{i}\right\rangle\right\rangle \in P_{n-m(i)} F$ (where $m(i) \leq k$ denotes the fold of $\rho_{i}$ ), we have that

$$
\pi \equiv \sum_{i=1}^{r} \tau_{i} \otimes \rho_{i} \quad\left(\bmod I^{n+1} F\right)
$$

Note that $n-m(i) \geq n-k \geq 2$. Thus, let $\tilde{\tau}_{i} \simeq\left\langle\left\langle 1, \ldots, 1, t_{i}\right\rangle\right\rangle \in P_{n-m(i)-1} F$ so that $\tau_{i} \simeq\left\langle\langle 1\rangle \otimes \otimes \tilde{\tau}_{i}\right.$. As $\tilde{u}(F) \leq 2^{\ell} \leq 2^{n-1}$, we have that $F$ is $(n-1)$-linked. Hence, there exists $\tilde{\pi} \in P_{n-1} F$ such that

$$
\tilde{\pi} \equiv \sum_{i=1}^{r} \tilde{\tau}_{i} \otimes \rho_{i} \quad\left(\bmod I^{n} F\right)
$$

We observe that $\tilde{\pi}_{K} \equiv 0 \quad\left(\bmod I^{n} K\right)$. Thus, by APH, $\tilde{\pi}_{K}=0$, i.e., $\tilde{\pi} \in P_{n-1} F \cap W(K / F)$. Now $\left.\operatorname{sgn}_{P}\left(\tau_{i} \otimes \rho_{i}\right)=\operatorname{sgn}_{P}(\langle 1\rangle\rangle \otimes \tilde{\tau}_{i} \otimes \rho_{i}\right)=2 \operatorname{sgn}_{P}\left(\tilde{\tau}_{i} \otimes \rho_{i}\right)$ for all $P \in X_{F}$, and one easily checks that therefore

$$
\operatorname{sgn}_{P} \pi=2 \operatorname{sgn}_{P} \tilde{\pi} \text { for all } P \in X_{F} .
$$


In particular, $\gamma$ is indefinite at $P$ (i.e., $1 \leq \operatorname{sgn}_{P} \gamma \leq 2^{n-1}-1$ ) iff $\operatorname{sgn}_{P} \pi=0$ iff $\operatorname{sgn}_{P} \tilde{\pi}=0$, and $\gamma$ is definite at $P$ (i.e., $\operatorname{sgn}_{P} \gamma=2^{n-1}+1$ ) iff $\operatorname{sgn}_{P} \pi=2^{n}$ iff $\operatorname{sgn}_{P} \tilde{\pi}=2^{n-1}$. It follows immediately that

$$
\left|\operatorname{sgn}_{P}(\gamma \perp-\tilde{\pi})\right| \leq 2^{n-1}-1 \text { for all } P \in X_{F},
$$

and since $\tilde{u}(F) \leq 2^{n-1}$ it follows from Lemma 2.1 that $\operatorname{dim}(\gamma \perp-\tilde{\pi})_{\text {an }} \leq 2^{n-1}-1$ (recall that $n \geq 2$ and thus $\operatorname{dim}(\gamma \perp-\tilde{\pi})$ is odd). Comparing this with the dimension of $\gamma \perp-\tilde{\pi}$ itself, we get for the Witt index $i_{W}(\gamma \perp-\tilde{\pi}) \geq 2^{n-2}+1$. It follows that $\gamma$ contains a Pfister neighbor $\tilde{\gamma}$ of $\tilde{\pi}$ with $\operatorname{dim} \tilde{\gamma}=2^{n-2}+1<\operatorname{dim} \gamma$. $\tilde{\gamma}_{K}$ is isotropic because $\tilde{\pi}_{K}=0$ and we conclude that $\gamma$ is not $K$-minimal.

To prove the remaining part of the theorem, let now $\left\{\pi_{\alpha}\right\} \cap P_{k} F=\{\rho\}$. Let $\pi \in P_{n} F \cap$ $W(K / F)$ and let $\gamma$ be a Pfister neighbor of $\pi$ with $\operatorname{dim} \gamma=2^{n-1}+1$. Again, we assume that $\operatorname{sgn}_{P} \gamma \geq 1$ for all $P \in X_{F}$. By a similar reasoning as above, in order to establish that $t_{\max }(K / F) \leq \max \left\{\tilde{u}(F), 2^{k-1}+1\right\}$, it suffices to show that if $n-1>\max \{k-1, \ell-1\}$ then $\gamma$ is not $K$-minimal (as before, $\ell$ is such that $\tilde{u}(F) \leq 2^{\ell}$ ).

So let us assume that $n-1>\max \{k-1, \ell-1\}$. In fact, if $n-1>k$ then the above proof of the first part of the theorem carries over without any changes. Hence, we assume from now on that $n-1=k$ and thus necessarily $k \geq \ell$. Again, let us write

$$
\pi \equiv \sum_{i=1}^{r} \tau_{i} \otimes \rho_{i} \quad\left(\bmod I^{n+1} F\right)
$$

with $\tau_{i}, \rho_{i}$, and also $t_{i}$ and $m(i)$ as above. If $m(i) \leq k-1$ for all $1 \leq i \leq r$ then the proof given above for the first part of the theorem also applies in this situation and we are done. So we may assume that at least one of the $\rho_{i}$ 's has fold $k$. By the proof of Lemma 3.1, we may also assume that the $\rho_{i}$ 's are pairwise non-isometric. Since there is only one $k$-fold Pfister form $\rho$ in $\left\{\pi_{\alpha}\right\}$, we may therefore assume that $\rho_{r} \simeq \rho, m(r)=k$, and $m(i) \leq k-1$ for $1 \leq i \leq r-1$. We put $t_{r}=t$ and get that $\tau_{r} \simeq\langle\langle t\rangle$ (recall that $n-1=k$ and thus $n-m(r)=n-k=1)$. We now define the following clopen sets in $X_{F}$ :

$$
\begin{aligned}
& Y=\left\{P \in X_{F} \mid \pi \text { is definite at } P\right\} \\
& Y_{1}=\left\{P \in Y \mid \rho \text { is definite at } P \text { and } t<_{P} 0\right\} \\
& Y_{2}=\left\{P \in Y \mid \rho \text { is definite at } P \text { and } t>_{P} 0\right\} \\
& Y_{3}=\{P \in Y \mid \rho \text { is indefinite at } P\} .
\end{aligned}
$$

Comparing signatures, one easily checks that

$$
\operatorname{sgn}_{P}\left\langle\langle t\rangle \otimes \rho= \begin{cases}0 & \text { if } P \in Y_{1} \cup Y_{3} \\ 2^{n} & \text { if } P \in Y_{2}\end{cases}\right.
$$

and

$$
\operatorname{sgn}_{P} \sum_{i=1}^{r-1} \tau_{i} \otimes \rho_{i} \equiv \begin{cases}2^{n}\left(\bmod 2^{n+1}\right) & \text { if } P \in Y_{1} \cup Y_{3} \\ 0 \quad\left(\bmod 2^{n+1}\right) & \text { if } P \in Y_{2} .\end{cases}
$$

Let $Z_{i}=\left\{P \in Y_{1} \mid t_{i}>_{P} 0\right\}$ for $1 \leq i \leq r-1$ and let $x_{i} \in \dot{F}$ such that $x_{i}<_{P} 0$ if $P \in Z_{i}$ and $x_{i}>_{P} 0$ if $P \notin Z_{i}$. Finally, let $\tilde{\tau}_{i} \simeq\left\langle\left\langle 1, \ldots, x_{i} t_{i}\right\rangle\right) \in P_{n-m(i)-1} F$ for $1 \leq i \leq r-1$. One readily concludes that for $1 \leq i \leq r-1$ we get

$$
\operatorname{sgn}_{P} \tilde{\tau}_{i} \otimes \rho_{i}= \begin{cases}0 & \text { if } P \in Y_{1} \\ \frac{1}{2} \operatorname{sgn}_{P} \tau_{i} \otimes \rho_{i} & \text { if } P \notin Y_{1}\end{cases}
$$


and furthermore

$$
\operatorname{sgn}_{P} \rho= \begin{cases}2^{n-1} & \text { if } P \in Y_{1} \\ \frac{1}{2} \operatorname{sgn}_{P}\langle\langle t\rangle\rangle \otimes \rho & \text { if } P \in Y \backslash Y_{1} .\end{cases}
$$

As $F$ is $(n-1)$-linked, there exists $\tilde{\pi} \in P_{n-1} F$ with

$$
\tilde{\pi} \equiv \sum_{i=1}^{r-1} \tilde{\tau}_{i} \otimes \rho_{i}+\rho \quad\left(\bmod I^{n} F\right) .
$$

We note that $\tilde{\pi} \in P_{n-1} F \cap W(K / F)$. Comparing signatures modulo $2^{n}$, one readily concludes that for all $P \in Y$ we have $\operatorname{sgn}_{P} \tilde{\pi}=\frac{1}{2} \operatorname{sgn}_{P} \pi=2^{n-1}=2^{k}$. Similarly as above, we see that $\operatorname{sgn}_{P} \gamma=2^{n-1}+1$ iff $\operatorname{sgn}_{P} \pi=2^{n}$, i.e., $\pi$ is definite at $P$, which by definition means that $P \in Y$ and thus $\operatorname{sgn}_{P} \tilde{\pi}=2^{n-1}$. As $\operatorname{sgn}_{P} \tilde{\pi} \in\left\{0,2^{n-1}\right\}$ and $1 \leq \operatorname{sgn}_{P} \gamma \leq 2^{n-1}-1$ for all $P \notin Y$, we get

$$
\left|\operatorname{sgn}_{P}(\gamma \perp-\tilde{\pi})\right| \leq 2^{n-1}-1 \text { for all } P \in X_{F} \text {. }
$$

Using the same reasoning as before, there exists a Pfister neighbor $\tilde{\gamma}$ of $\tilde{\pi}$ of dimension $2^{n-2}+1$ with $\tilde{\gamma} \subset \gamma . \tilde{\gamma}_{K}$ is isotropic because $\tilde{\pi}_{K}=0$, and $\operatorname{dim} \tilde{\gamma}=2^{n-2}+1<\operatorname{dim} \gamma=$ $2^{n-1}+1$, which yields that $\gamma$ is not $K$-minimal.

Corollary 5.4 Let $F$ be linked and $\pi \in P_{n} F$ be anisotropic. Let $K=F(\pi)$. Then the $K$-minimal forms are exactly the Pfister neighbors of $\pi$ of dimension $2^{n-1}+1$.

Proof. The case $n=1$ has been mentioned before (and we don't need $F$ being linked in this case). The case $n=2$ was first proved in [H 1 , Theorem 4.5.2], a simplified proof can be found in [HLVG, Prop. 4.4]. If $n>3$ the statement follows from the fact that if $F$ is linked then $\tilde{u}(F) \leq 8$ and by invoking Theorems 5.1 and 5.3. Finally, if $n=3$ then Theorem 5.3 implies that any $K$-minimal form has dimension $\leq 8$. However, any anisotropic form over $F$ of dimension $\leq 8$ which becomes isotropic over $K$ contains a Pfister neighbor of $\pi$ of dimension 5 by Proposition 4.7, which completes the proof.

\section{Characterization of $K$-minimal forms}

In the previous section, we have provided certain bounds for the dimensions of $K$-minimal forms for Pfister extensions $K / F$ and found an exact lower bound in all cases, and upper bounds under the assumption that $\tilde{u}(F)<\infty$. We now will give a precise description of $K$-minimal forms for Pfister extensions $K / F$ where $\tilde{u}(F)<\infty$ and where the Pfister forms involved are of "suitably" large dimension.

It follows easily from the definition of $K$-minimal forms that anisotropic Pfister forms of fold $\geq 2$ can never be $K$-minimal for any field extension $K / F$. This is simply because if $\pi_{K}$ is isotropic then $\pi_{K}$ is hyperbolic and any subform of $\pi$ of dimension $2^{n-1}+1<2^{n}=\operatorname{dim} \pi$ will be isotropic over $K$. Nevertheless, we will define a version of $K$-minimality for Pfister forms which will be useful later on.

Definition 6.1 Let $K / F$ be a field extension. Then $\pi \in P_{n} F, n \geq 1$, is a Pfister $K$ minimal form if the following three conditions are fulfilled:

(i) $\pi$ is anisotropic over $F$.

(ii) $\pi_{K}$ is isotropic and hence hyperbolic, i.e., $\pi \in W(K / F)$.

(iii) For any $\sigma \in P_{m} F$ with $\sigma \subset \pi$ and $m<n$ we have that $\sigma_{K}$ is anisotropic. 
One easily sees that to check whether condition (iii) holds it suffices to check that it holds for $m=n-1$. In this case $(m=n-1)$ and with $\sigma$ and $\pi$ as above, there exists $t \in \dot{F}$ such that $\pi \simeq \sigma \otimes\langle\langle t\rangle\rangle$.

We would like to describe the $K$-minimal forms for some of the Pfister extensions $K / F$ dealt with in the previous sections. It turns out that they will be Pfister neighbors. Indeed, they will be Pfister neighbors of Pfister $K$-minimal forms. The following lemma relates $K$-minimal forms which are Pfister neighbors and Pfister $K$-minimal forms. The assumptions are in a way so that they apply directly to the cases we consider.

Lemma 6.2 Let $F$ be formally real with $\tilde{u}(F) \leq 2^{n}$. Let $K / F$ be a field extension such that $K$-minimal forms are always Pfister neighbors. Let $\pi \in P_{n+1} F \cap W(K / F)$ be anisotropic and let $\varphi$ be a Pfister neighbor of $\pi$. Then the following statements are equivalent.

(i) $\varphi$ is $K$-minimal.

(ii) $\operatorname{dim} \varphi=2^{n}+1$ and $\pi$ is Pfister $K$-minimal.

Proof. The case $n=0$ is trivial. So let $n \geq 1$. Also, we may assume from the beginning that $\operatorname{dim} \varphi=2^{n}+1$. For if a Pfister neighbor $\varphi$ of the $(n+1)$-Pfister form $\pi$ is isotropic, then $\pi$ is hyperbolic and any subform of $\varphi$ of dimension $2^{n}+1$ is isotropic as well.

(i) $\Rightarrow$ (ii). Suppose $\pi$ is not Pfister $K$-minimal. Then there exist $\sigma \in P_{n} F, t \in \dot{F}$, such that $\sigma_{K}=0$ and $\pi \simeq \sigma \otimes\left\langle\langle t\rangle\right.$. Let $Y=\left\{P \in X_{F} \mid \operatorname{sgn}_{P} \sigma=0\right\}$ and $Z=\{P \in$ $\left.X_{F} \mid \operatorname{sgn}_{P} \pi=0\right\}$. Note that $Y \subset Z$, and that $\operatorname{sgn}_{P} \sigma=2^{n}$ for all $P \in X_{F} \backslash Y$ and $\operatorname{sgn}_{P} \pi=2^{n+1}$ for all $P \in X_{F} \backslash Z$. As $\tilde{u}(F)<\infty, F$ is SAP and we may assume that after possibly scaling, we have $\operatorname{sgn}_{P} \varphi \geq 1$ for all $P \in X_{F}$ (note that $\operatorname{dim} \varphi$ is odd). In particular, $\operatorname{sgn}_{P} \varphi=2^{n}+1$ for all $P \in X_{F} \backslash Z$ and $1 \leq \operatorname{sgn}_{P} \varphi \leq 2^{n}-1$ for all $P \in Z$. We get

$$
\left|\operatorname{sgn}_{P}(\varphi \perp-\sigma)\right| \leq 2^{n}-1 \text { for all } P \in X_{F} .
$$

Hence, by Lemma 2.1 and with $\tilde{u}(F) \leq 2^{n}$, it follows that $\operatorname{dim}(\varphi \perp-\sigma)_{\text {an }} \leq 2^{n}-1$ (note that $\operatorname{dim}(\varphi \perp-\sigma)_{\text {an }}$ is odd $)$. By comparing dimensions, we conclude that $i_{W}(\varphi \perp-\sigma) \geq$ $2^{n-1}+1$. Therefore, there exists $\chi \subset \sigma$ with $\operatorname{dim} \chi=2^{n-1}+1$ such that $\chi \subset \varphi$. In particular, $\chi$ is a Pfister neighbor of $\sigma$, and because $\sigma_{K}=0$ we have that $\chi_{K}$ is isotropic. But $\chi \subset \varphi$ and $\operatorname{dim} \chi \leq \operatorname{dim} \sigma<\operatorname{dim} \varphi$ which implies that $\varphi$ is not $K$-minimal.

(ii) $\Rightarrow$ (i). As $\pi_{K}=0$ we have that $\varphi_{K}$ is isotropic. If $\varphi$ is not $K$-minimal then there exists a $K$-minimal form $\chi \subset \varphi$ with $\operatorname{dim} \chi<\operatorname{dim} \varphi=2^{n}+1$. By assumption, $\chi$ itself is a Pfister neighbor. Thus, $\chi$ is a Pfister neighbor of some $\tau \in P_{m} F$ with $m \leq n$. Then $\chi_{F(\tau)}$ is isotropic, hence also $\varphi_{F(\tau)}$ and therefore $\pi_{F(\tau)}$ is hyperbolic. We readily conclude that by the Cassels-Pfister subform theorem, $\tau \subset \pi$. Also, $\chi_{K}$ is isotropic and thus $\tau_{K}$ is hyperbolic. In particular, $\pi$ is not Pfister $K$-minimal.

It should be noted that the assumption on $F$ being formally real with $\tilde{u}(F) \leq 2^{n}$ was only needed to show (i) $\Rightarrow$ (ii), and that the assumption on $K$-minimal forms being Pfister neighbors was only used to show (ii) $\Rightarrow$ (i).

Remark 6.3 There are examples of field extensions $K / F$ where there exists a Pfister $K$ minimal form $\pi$ which contains $K$-minimal forms of dimension $\leq \frac{1}{2} \operatorname{dim} \pi$. For instance, let $F$ be any field with an anisotropic 3-fold Pfister form $\pi$ such that $\pi$ contains a subform $\psi$ with $\operatorname{dim} \psi=4$ and $\psi \notin G P_{2} F$. Let $K=F(\psi)$. The only anisotropic forms of dimension $\leq 4$ over $F$ which become isotropic over $K$ are exactly the forms similar to $\psi$ (see, e.g., [H 2]). It follows that $\psi$, which is not a Pfister neighbor, is $K$-minimal, and that $\pi$ is 
Pfister $K$-minimal. (ii) $\not$ (i) in this case because no 5 -dimensional subform of $\pi$ containing $\psi$ will be $K$-minimal. Note that in this example, $K / F$ is not excellent because $\psi$ is not a Pfister neighbor.

Remark 6.4 If all $K$-minimal forms are Pfister neighbors then one readily sees that $K / F$ is excellent. However, the converse is not true in general. In [HVG], there is an example of a field $F$ with an anisotropic conic $\rho \simeq\langle 1, a, b\rangle$ over $F$ such that for $K=F(\rho)$ one has $t_{\max }(K / F)=\infty$. In particular, by [HLVG, Corollary 3.4], there exist 7-dimensional $K$-minimal forms. Such a form cannot be a Pfister neighbor because if $\varphi$ is an anisotropic 7-dimensional Pfister neighbor over $F$ with $\varphi_{K}$ isotropic then it is an easy excercise to show that $\varphi$ contains a subform similar to $\rho$ and is therefore not $K$-minimal. Hence, there exist (7-dimensional) $K$-minimal forms which are not Pfister neighbors. On the other hand, $K / F$ is known to be excellent (see the introduction).

For the remainder of this section, we fix the following notations.

$$
\begin{aligned}
& m \quad \text { an integer } \geq 1 \\
& F \quad \text { a field (char } F \neq 2 \text { ) which fulfills the following: } \\
& \text { If } m=1 \text { or } 2 \text { then } \tilde{u}(F) \leq 2^{m} \text {. } \\
& \text { If } m=3 \quad \text { then } \quad \tilde{u}(F) \leq 6 \text { or } \tilde{u}(F)=8 \text { and } F \text { linked. } \\
& \text { If } m \geq 4 \quad \text { then } \tilde{u}(F) \leq 2^{m-1}
\end{aligned}
$$

Theorem 6.5 With the notations as above, let $K=F\left(\left\{\rho_{i}^{(n)}\right\}, n \geq m\right)$. Let $\varphi \in W F$ be anisotropic. Then the following statements are equivalent.

(i) $\varphi$ is $K$-minimal.

(ii) There exist $n \geq m$ and $\pi \in \Phi_{n} \backslash \Phi_{n}^{\prime}$ such that $\varphi$ is a Pfister neighbor of dimension $2^{n-1}+1$ of $\pi$.

Proof. It follows from Lemma 3.2 and Proposition 4.7 (see also Remark 5.2 in the case $m=2$ ), that any anisotropic form over $F$ which becomes isotropic over $K$ already contains a Pfister neighbor which becomes isotropic over $K$. In particular, $K$-minimal forms will always be Pfister neighbors. Now if $\varphi$ is a Pfister neighbor of some $n$-fold Pfister form $\pi$ and if $\varphi$ is $K$-minimal, then clearly $\operatorname{dim} \varphi=2^{n-1}+1$ and necessarily $n \geq m$ by the Anisotropy Criterion. Thus, $K$-minimal forms are Pfister neighbors of dimension $2^{n-1}+1$ where $n \geq m$.

The $K$-minimal forms of dimension $2^{m-1}+1$ are exactly the Pfister neighbors of dimension $2^{m-1}+1$ of anisotropic Pfister forms in $\Phi_{m}$ (i.e., Pfister forms in $\Phi_{m} \backslash \Phi_{m}^{\prime}$ ) by Theorem 5.1. 
It remains to determine those Pfister neighbors of dimension $2^{n-1}+1, n>m$, which are $K$-minimal. Note that $\tilde{u}(F) \leq 2^{m} \leq 2^{n-1}$. By Lemma 6.2 , these are exactly the Pfister neighbors of dimension $2^{n-1}+1$ of $n$-fold Pfister $K$-minimal forms. It therefore suffices to determine Pfister $K$-minimal forms of dimension $2^{n}, n>m$. We have to show that $\pi$ is Pfister $K$-minimal of dimension $2^{n}$ iff $\pi \in \Phi_{n} \backslash \Phi_{n}^{\prime}$.

One easily shows by induction and by invoking LGT that $\Phi_{n} \subset W(K / F)$. Now let $\pi \in P_{n} F \cap W(K / F)$. It follows immediately from Lemma 3.2 that $\pi \in \Phi_{n}$. Thus, $P_{n} F \cap W(K / F)=\Phi_{n}$. (This also holds for $m$ because by LGT, $\Phi_{m}=P_{m} F \cap W(K / F)$.) Suppose that $\pi \in \Phi_{n}$ is anisotropic but not Pfister $K$-minimal. Then, by definition of Pfister $K$-minimality, there exist $t \in \dot{F}$ and $\tau \in P_{n-1} F \cap W(K / F)$ such that $\pi \simeq\langle\langle t\rangle \otimes \tau$. By the above, $\tau \in \Phi_{n-1}$. Thus, $\pi \in \Phi_{n}^{\prime}$. Conversely, if $\pi \in \Phi_{n}^{\prime}$ then $\pi \simeq\langle\langle t\rangle \otimes \tau$ for some $t \in \dot{F}$ and $\tau \in \Phi_{n-1}$. But then $\tau \in P_{n-1} F \cap W(K / F)$ which implies that $\pi$ is not Pfister $K$-minimal.

This shows that for all $n \geq m$, the set of Pfister $K$-minimal forms of dimension $2^{n}$ is exactly the set $\Phi_{n} \backslash \Phi_{n}^{\prime}$, and that therefore the $K$-minimal forms are exactly the Pfister neighbors of dimension $2^{n-1}+1, n \geq m$, of Pfister forms in $\Phi_{n} \backslash \Phi_{n}^{\prime}$.

There is an alternative description of $K$-minimal resp. Pfister $K$-minimal forms which uses properties of $\boldsymbol{X}_{\boldsymbol{F}}$.

The clopen sets in any topological space $T$ form an abelian group of exponent 2 with addition defined as follows:

$$
U+V=(U \cup V) \backslash(U \cap V), \quad U, V \text { clopen. }
$$

Let $F$ be formally real. To each $\varphi \in W F$ we associate a clopen set $Y(\varphi) \subset X_{F}$ by

$$
Y(\varphi)=\left\{P \in X_{F} \mid \varphi \text { is definite at } P\right\} .
$$

One easily checks that $Y(\varphi \otimes \psi)=Y(\varphi) \cap Y(\psi)$, and that if $\varphi$ is a Pfister neighbor of the Pfister form $\pi$ then $Y(\varphi)=Y(\pi)$. It is also rather obvious that if $\pi_{1}, \pi_{2} \in P_{n} F$ are linked with $\pi \equiv \pi_{1}+\pi_{2} \quad\left(\bmod I^{n+1} F\right)$, where $\pi \in P_{n} F$, then

$$
Y(\pi)=Y\left(\pi_{1}\right)+Y\left(\pi_{2}\right) .
$$

Let $\mathcal{X}_{F}^{\text {clop }}$ be the abelian group of clopen sets in $X_{F}$ with addition defined as above. Suppose furthermore that $\Phi_{n} \subset P_{n} F$ is a linked group of $n$-fold Pfister forms. Then equation (2) immediately yields a group homomorphism

$$
\Gamma: \Phi_{n} \longrightarrow \mathcal{X}_{F}^{\text {clop }}: \pi \longmapsto Y(\pi) .
$$

If $I_{t}^{n} F=0$ then $\Gamma$ is injective because one readily sees that in this case two $n$-fold Pfister forms are isometric iff they have the same total signatures. Let

Let $F, m \geq 1,\left\{\rho_{i}^{(n)}\right\}, \Phi_{n}, \Phi_{n}^{\prime}, n \geq m$, be as defined at the beginning of this section.

$$
\begin{aligned}
& \mathcal{T}_{n}=\left\{Y(\pi) \mid \pi \in \Phi_{n}\right\} \subset \mathcal{X}_{F}^{\text {clop }} \\
& \mathcal{T}_{n}^{\prime}=\left\{Y(\pi) \mid \pi \in \Phi_{n}^{\prime}\right\} \subset \mathcal{X}_{F}^{\text {clop }}
\end{aligned}
$$

If $I_{t}^{n} F=0$ then by definition, $\mathcal{T}_{n}$ is a subgroup of $\mathcal{X}_{F}^{\text {clop }}$ isomorphic to $\Phi_{n}$, and there is bijection between $\mathcal{T}_{n}^{\prime}$ and $\Phi_{n}^{\prime}$ (both bijections are induced by $\Gamma$ ). Recall that we assumed $\tilde{u}(F)<\infty$ so that $F$ is in fact SAP. This implies that to each clopen $Y \subset X_{F}$ there exists $t \in \dot{F}$ such that $Y=Y(\langle t\rangle\rangle)$. Recall also that $\Phi_{n}^{\prime}=\left\{\pi \otimes\langle\langle t\rangle| \pi \in \Phi_{n-1}, t \in \dot{F}\right\}$. 
Using the fact that $Y\left(\pi \otimes\langle\langle t\rangle)=Y(\pi) \cap Y\left(\langle\langle t\rangle)\right.\right.$, we conclude that if $2^{n}>\tilde{u}(F)$ then $\mathcal{T}_{n}^{\prime}=\left\{Y_{1} \cap Y_{2} \mid Y_{1} \in \mathcal{T}_{n-1}, Y_{2} \in \mathcal{X}_{F}^{\text {clop }}\right\}$, and $\mathcal{T}_{n}$ is the subgroup of $\mathcal{X}_{F}^{\text {clop }}$ generated by $\left\{Y(\pi), \pi \in\left\{\rho_{i}^{(n)}\right\}\right\} \cup \mathcal{T}_{n}^{\prime}$.

If $n>m$ then $2^{n-1}+1>\tilde{u}(F)$ by the definition of $m$. In this case, it follows that if $\varphi$ is a form over $F$ of dimension $2^{n-1}+1$ then $\varphi$ is a Pfister neighbor (see [F, Prop. 4.7]), say, of $\pi \in P_{n} F$. So if $\varphi$ is anisotropic then $\varphi_{K}$ is isotropic iff $\pi \in \Phi_{n}$, and $\varphi$ is $K$-minimal iff $\pi \in \Phi_{n} \backslash \Phi_{n}^{\prime}$. Using the fact that $Y(\varphi)=Y(\pi)$, we now get the following alternative version of Theorem 6.5 .

Theorem 6.6 Let $m \geq 1, F, K, \Phi_{m}, \mathcal{T}_{n}, \mathcal{T}_{n}^{\prime}, n \geq m$, be as above. Let $\varphi \in W F$. Then $\varphi$ is $K$-minimal if and only if either

(i) $\operatorname{dim} \varphi=2^{m-1}+1$ and $\varphi$ is a Pfister neighbor of some anisotropic $\pi \in \Phi_{m}$, or

(ii) $\operatorname{dim} \varphi=2^{n-1}+1$ with $n>m$ and $Y(\varphi) \in \mathcal{T}_{n} \backslash \mathcal{T}_{n}^{\prime}$.

Furthermore, if $\tilde{u}(F) \leq 2^{m-1}$ then the $K$-minimal forms of dimension $2^{m-1}+1$ are exactly the forms $\varphi$ of dimension $2^{m-1}+1$ with $Y(\varphi) \in \mathcal{T}_{m} \backslash\{\theta\}$.

\section{Two examples}

Example 7.1 Consider the global field $F=\mathbf{Q}(\sqrt{2}, \sqrt{3})$. We have $\tilde{u}(F)=4$. There are four orderings on $F, X_{F}=\{P, Q, R, S\}$, which correspond to the four embeddings of $F$ into the real numbers $\mathbf{R}$ as follows. Let $x=x_{1}+x_{2} \sqrt{2}+x_{3} \sqrt{3}+x_{4} \sqrt{6}$ with $x_{i} \in \mathbf{Q}$. Then

$$
\begin{aligned}
& P: x \longmapsto x_{1}+x_{2} \sqrt{2}+x_{3} \sqrt{3}+x_{4} \sqrt{6} \\
& Q: x \longmapsto x_{1}-x_{2} \sqrt{2}+x_{3} \sqrt{3}-x_{4} \sqrt{6} \\
& R: x \longmapsto x_{1}+x_{2} \sqrt{2}-x_{3} \sqrt{3}-x_{4} \sqrt{6} \\
& S: x \longmapsto x_{1}-x_{2} \sqrt{2}-x_{3} \sqrt{3}+x_{4} \sqrt{6}
\end{aligned}
$$

Let

$$
a_{0}=-1, a_{1}=\sqrt{2}+\sqrt{3}-\sqrt{6}, a_{2}=-\sqrt{2}+\sqrt{3}+\sqrt{6}, a_{3}=-a_{1} a_{2}=5-4 \sqrt{3}
$$

and

$$
b_{1}=\sqrt{2}-\sqrt{3}+\sqrt{6}, b_{2}=-\sqrt{2}-\sqrt{3}-\sqrt{6}, b_{3}=1 .
$$

Let $m \geq 2$. We now define $m$-fold and $(m+1)$-fold Pfister forms as follows :

$$
\begin{array}{ll}
\alpha_{i} \simeq\left\langle\left\langle 1, \cdots, 1, a_{i}\right\rangle\right\rangle \in P_{m} F & i=0,1,2,3 \\
\beta_{i} \simeq\left\langle\left\langle 1, \cdots, 1, b_{i}\right\rangle\right\rangle \in P_{m+1} F & i=1,2,3
\end{array}
$$

Let $K=F\left(\alpha_{1}, \alpha_{2}\right)$. Then $\left\{\alpha_{1}, \alpha_{2}\right\}$ generates the linked group $\Phi_{m}=\left\{\alpha_{0}, \alpha_{1}, \alpha_{2}, \alpha_{3}\right\}$ of $m$-fold Pfister forms (note that $\alpha_{0}=0$ ), and one easily checks that with the notations as at the end of the previous section, we have

$$
\mathcal{T}_{m}=\left\{Y\left(\alpha_{0}\right), Y\left(\alpha_{1}\right), Y\left(\alpha_{2}\right), Y\left(\alpha_{3}\right)\right\}=\{\emptyset,\{P, Q, R\},\{P, Q, S\},\{R, S\}\} .
$$

We find that $\mathcal{T}_{m+1}^{\prime}$ consists of all subsets of $X_{F}$ other than those of cardinality $\geq 3$ which contain both $R$ and $S$, i.e.,

$$
\mathcal{T}_{m+1}^{\prime}=\mathcal{X}_{F}^{\text {clop }} \backslash\{\{P, R, S\},\{Q, R, S\},\{P, Q, R, S\}\} .
$$


Note that $\mathcal{T}_{m+1}^{\prime}=\left\{Z \mid \exists Y \in \mathcal{T}_{m}: Z \subset Y\right\} . \mathcal{T}_{m+1}$ is the group generated by $\mathcal{T}_{m+1}^{\prime}$, and one easily checks that $\mathcal{T}_{m+1}=\mathcal{X}_{F}^{\text {clop }}$. Thus,

$$
\mathcal{T}_{m+1} \backslash \mathcal{T}_{m+1}^{\prime}=\{\{P, R, S\},\{Q, R, S\},\{P, Q, R, S\}\}=\left\{Y\left(\beta_{1}\right), Y\left(\beta_{2}\right), Y\left(\beta_{3}\right)\right\} .
$$

Theorems 5.3 and 6.6 now yield the following result.

Proposition 7.2 With the same notations as above, the $K$-minimal forms are exactly the Pfister neighbors of dimension $2^{m-1}+1$ of the $\alpha_{i}$ 's, $i=1,2,3$, and the Pfister neighbors of dimension $2^{m}+1$ of the $\beta_{i}$ 's, $i=1,2,3$.

Example 7.3 Let $F=\mathbf{R}(t)$. It is well-known that $\tilde{u}(F)=2$. Furthermore, the orderings on $F$ can be described as follows (see [KS, Ch. II, $\S 9$, Satz 1]). To each $c \in \mathbf{R} \cup\{\infty\}$ there exist two orderings $P_{c_{1}+}$ and $P_{c_{1}-}$, and for $f \in \mathbf{R}(t)$ we have that

$$
\begin{array}{ll}
f>P_{c,+} 0 & \text { if } \exists \epsilon>0: f(x)>0 \forall c<x<c+\epsilon, \text { where } c \in \mathbf{R} \\
f>P_{c_{1}-} 0 & \text { if } \exists \epsilon>0: f(x)>0 \forall c-\epsilon<x<c \text {, where } c \in \mathbf{R} \\
f>P_{\infty,+} 0 & \text { if } f(x)>0 \text { for all } x \gg 0 \\
f>P_{\infty,-} 0 & \text { if } f(x)>0 \text { for all } x \ll 0
\end{array}
$$

For convenience, let us define the following "intervals" of orderings in $X_{F}$ :

$$
(a, b)=\left\{P_{c,+}, P_{c,-}, P_{a,+}, P_{b,-} ; a<c<b\right\} \text {, where } a<b \text { in } \mathbf{R} .
$$

For $a=-\infty$ we replace $P_{a,+}$ by $P_{\infty,-}$, and for $b=+\infty$ we replace $P_{b,-}$ by $P_{\infty,+}$. If $0 \neq f(t)=\frac{g(t)}{h(t)}$, where $g(t), h(t) \in \mathbf{R}[t]$, then $f>_{P} 0$ for all $P \in(a, b)$ if and only if $g(x) h(x) \geq 0$ for all $a<x<b$. Every clopen set in $X_{F}$ is just a finite union of intervals $(a, b)$.

For each $n \geq 1$, we now define an anisotropic $n$-fold Pfister form $\beta_{n}$ over $F$ by $\beta_{n} \simeq$ $\langle\langle 1, \cdots, 1, n-t\rangle\rangle$, and we let $K=F\left(\beta_{1}, \beta_{2}, \cdots\right)$. We have that

$$
Y\left(\beta_{n}\right)=\left\{P \in X_{F} \mid \beta_{n} \text { is positive definite at } P\right\}=(-\infty, n) .
$$

With this, we conclude that for $n \geq 2$ we get

$$
\begin{aligned}
& \mathcal{T}_{n}^{\prime}=\left\{Y \subset X_{F} \text { clopen } \mid Y \subset(-\infty, n-1)\right\} \\
& \mathcal{T}_{n}=\{Y, Y \cup(n-1, n) \mid Y \subset(-\infty, n-1) \text { clopen }\} .
\end{aligned}
$$

We know by Theorems 6.5 resp. 6.6, that the $K$-minimal forms are Pfister neighbors of dimension $2^{n-1}+1$. For $n=1$, these theorems imply that the $K$-minimal forms are exactly those forms similar to $\beta_{1} \simeq\langle\langle 1-t\rangle\rangle$. For $n \geq 2$, we recall that $\tilde{u}(F)=2$ and that therefore, by these theorems, the $K$-minimal forms are exactly the Pfister neighbors of dimension $2^{n-1}+1$ of Pfister forms $\beta$ with $Y(\beta) \in \mathcal{T}_{n} \backslash \mathcal{T}_{n}^{\prime}$. However, $\tilde{u}(F)=2$ and thus $F$ is SAP and $I_{t}^{n} F=0$ for $n \geq 2$. Hence, $n$-fold Pfister forms are uniquely determined by their total signatures and thus, every $\beta \in P_{n} F$ with $n \geq 2$ can be written in the form $\left\langle\langle 1, \cdots, 1, f\rangle\right.$ for some non-zero polynomial $f \in \mathbf{R}[t]$. For such a $\beta$ we have $Y(\beta) \in \mathcal{T}_{n} \backslash \mathcal{T}_{n}^{\prime}$ if and only if $f(x) \leq 0$ for all $x>n$ and $f(x) \geq 0$ for all $n-1<x<n$. We can summarize our results as follows.

Proposition 7.4 With the same notations as above, the $K$-minimal forms of dimension 2 are exactly the forms similar to $\beta_{1} \simeq\langle\langle 1-t\rangle\rangle$, and the $K$-minimal forms of dimension $2^{n-1}+1, n \geq 2$, are exactly the Pfister neighbors of dimension $2^{n-1}+1$ of $n$-fold Pfister forms $\langle\langle 1, \cdots, 1, f\rangle$, where $f$ is a non-zero polynomial with $f(x) \leq 0$ for all $x>n$ and $f(x) \geq 0$ for all $n-1<x<n$. In particular, all Pfister neighbors of $\beta_{n}$ of dimension $2^{n-1}+1$ are $K$-minimal (for example, $2^{n-1}\langle 1\rangle \perp\langle n-t\rangle$ ), and we have $t_{\max }(K / F)=\infty$. 
We can describe the $K$-minimal forms of dimension $m+1=2^{n-1}+1, n \geq 2$, in an alternative way. Recall that any form $\varphi$ of dimension $m+1=2^{n-1}+1, n \geq 2$, is a Pfister neighbor because $\tilde{u}(F)=2$. Furthermore, any such form can be written as $\varphi \simeq g\left\langle 1, f_{1}, \cdots, f_{m}\right\rangle$ with $0 \neq g, f_{i} \in \mathbf{R}[t], 1 \leq i \leq m$. Also, such a form $\varphi$ is $K$-minimal iff it is a Pfister neighbor of some $n$-fold Pfister form $\beta$ as above iff it is definite for all $P \in(n-1, n)$ and indefinite for all $P \in(n,+\infty)$. If we translate these (in)definiteness conditions into evaluating the $f_{i}$ 's at certain values $t=x$, we get :

Proposition 7.5 The form $\varphi \simeq g\left\langle 1, f_{1}, \cdots, f_{m}\right\rangle, m=2^{n-1} \geq 2, g, f_{i} \in \mathbf{R}[t]$, is $K$ minimal if and only if $f_{i}(x) \geq 0$ for all $n-1<x<n$ and all $1 \leq i \leq m$, and to each $x>n$ and $\epsilon>0$ there exist $i \in\{1, \cdots, m\}$ and $y$ with $|x-y|<\epsilon$ such that $f_{i}(y)<0$.

\section{References}

[AK] Arason, J.K.; Knebusch, M.: Über die Grade quadratischer Formen. Math. Ann. 234 (1978) 167-192.

[E] Elman, R.: Quadratic forms and the u-invariant, III. Proc. of Quadratic Forms Conference (ed. G. Orzech). Queen's Papers in Pure and Applied Mathematics No. 46 (1977) 422-444.

[EL 1] Elman, R.; LAM, T.Y.: Pfister forms and K-theory of fields. J. Algebra 23 (1972) 181-213.

[EL 2] Elman, R.; LAM, T.Y.: Quadratic forms and the u-invariant II. Invent. Math. 21 (1973) 125-137.

[ELP] Elman, R.; Lam, T.Y.; Prestel, A.: On some Hasse principles over formally real fields. Math. Z. 134 (1973) 291-301.

[ELW 1] Elman, R.; LAm, T.Y.; Wadsworth, A.R.: Amenable fields and Pfister extensions. Proc. of Quadratic Forms Conference (ed. G. Orzech). Queen's Papers in Pure and Applied Mathematics No. 46 (1977) 445-491.

[ELW 2] Elman, R.; LaM, T.Y.; WadsWorTh, A.R.: Orderings under field extensions. J. Reine Angew. Math. 306 (1979) 7-27.

[ELW 3] Elman, R.; LAM, T.Y.; WADsworTh, A.R.: Function fields of Pfister forms. Invent. Math. 51 (1979) 61-75.

[ELTW] Elman, R.; LAM, T.Y.; Tignol, J.-P.; WadsworTh, A.R.: Witt rings and Brauer groups under multiquadratic extensions, I. American J. Math. 105 (1983) 1119-1170.

[F] Fitzgerald, R.W.: Witt kernels of function field extensions. Pacific J. Math. 109 (1983) 89-106.

[H 1] Hoffmann, D.W.: Function Fields of Quadratic Forms. Doctoral Dissertation. University of California, Berkeley, California 1992.

[H 2] HoFFMANN, D.W.: Isotropy of 5-dimensional quadratic forms isotropic over the function field of a quadric. K-Theory and Algebraic Geometry: Connections with Quadratic Forms and Division Algebras, Proceedings of the 1992 Santa Barbara Summer Research Institute (eds. B. Jacob, A. Rosenberg). Proc. Symp. Pure Math. 58.2 (1995) 217-225.

[H 3] HofFMANN, D.W.: On 6-dimensional quadratic forms isotropic over the function field of a quadric. Comm. Alg. 22 (1994) 1999-2014.

[H 4] HoffmanN, D.W.: Isotropy of quadratic forms over the function field of a quadric. Math. Z. 220 (1995) 461-476.

[H 5] HoffmanN, D.W.: On quadratic forms of height 2 and a theorem of Wadsworth. To appear in: Trans. Amer. Math. Soc. 
[H 6] Hoffmann, D.W.: Twisted Pfister forms. Doc. Math. J. DMV 1 (1996) 67-102.

[HLVG] HoffmanN, D.W.; LEWIS, D.W.; VAN GeEL, J.: Minimal forms for function fields of conics. $K$-Theory and Algebraic Geometry: Connections with Quadratic Forms and Division Algebras, Proceedings of the 1992 Santa Barbara Summer Research Institute (eds. B. Jacob, A. Rosenberg). Proc. Symp. Pure Math. 58.2 (1995) 227-237.

[HVG] HoffmanN, D.W.; VAN GeEL, J.: Minimal forms with respect to function fields of conics. Manuscripta Math. 86 (1995) 23-48.

[I] Izhboldin, O.T.: On the nonexcellence of field extensions $F(\pi) / F$. Doc. Math. J. DMV 1 (1996) 127-136.

[Ka] KAHN, B.: Formes quadratiques de hauteur et de degré 2. Indag. Math. 7 (1996) 47-66.

[K 1] KNebusch, M.: Generic splitting of quadratic forms I. Proc. London Math. Soc. 33 (1976) 65-93.

[K 2] KNebusch, M.: Generic splitting of quadratic forms II. Proc. London Math. Soc. 34 (1977) 1-31.

[KS] KNebusch, M.; Scheiderer, C.: Einführung in die reelle Algebra. Braunschweig, Wiesbaden: Vieweg 1989.

[Lag] LAghrIBI, A.: Isotropie de certaines formes quadratiques de dimension 7 et 8 sur le corps des fonctions d'une quadrique. To appear in: Duke Math. J.

[L 1] LAM, T.Y.: The Algebraic Theory of Quadratic Forms. Reading, Massachusetts: Benjamin 1973 (revised printing 1980).

[L 2] LAM, T.Y.: Orderings, Valuations and Quadratic Forms. Regional Conference Series in Mathematics 52. Providence, Rhode Island: AMS 1983.

[L 3] LAM, T.Y.: Fields of u-invariant 6 after A. Merkurjev. Israel Math. Conf. Proc. Vol. 1: Ring Theory 1989 (in honor of S.A. Amitsur) (ed. L. Rowen). pp.12-31. Jerusalem: Weizmann Science Press 1989.

[M] MERKurJev, A.S.: Simple algebras and quadratic forms. Izv. Akad. Nauk. SSSR 55 (1991) 218-224. (English translation: Math. USSR Izvestiya 38 (1992) 215-221.)

[S] Scharlau, W.: Quadratic and Hermitian Forms. Berlin, Heidelberg, New York, Tokyo: Springer 1985.

[W] WADSWORTH, A.R.: Similarity of quadratic forms and isomorphisms of their function fields. Trans. Amer. Math. Soc. 208 (1975) 352-358.

Laboratoire de Mathématiques

Faculté des Sciences

Université de Franche-Comté

F-25030 Besançon Cedex

France

email: detlevemath.univ-fcomte.fr 